hep-th/9510101, IASSNS-HEP-95-78

\title{
SUPERSYMMETRIC YANG-MILLS THEORY AND INTEGRABLE SYSTEMS
}

\author{
Ron Donagi回 \\ Department of Mathematics, University of Pennsylvania \\ Philadelphia, PA 19104-6395, USA

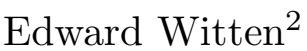 \\ School of Natural Sciences, Institute for Advanced Study \\ Olden Lane, Princeton, NJ 08540, USA
}

The Coulomb branch of $N=2$ supersymmetric gauge theories in four dimensions is described in general by an integrable Hamiltonian system in the holomorphic sense. A natural construction of such systems comes from two-dimensional gauge theory and spectral curves. Starting from this point of view, we propose an integrable system relevant to the $N=2 S U(n)$ gauge theory with a hypermultiplet in the adjoint representation, and offer much evidence that it is correct. The model has an $S L(2, \mathbf{Z}) S$-duality group (with the central element -1 of $S L(2, \mathbf{Z})$ acting as charge conjugation); $S L(2, \mathbf{Z})$ permutes the Higgs, confining, and oblique confining phases in the expected fashion. We also study more exotic phases.

October, 1995

1 Research supported in part by NSF grant DMS95-03249 and Unite mixte de service de l'institut H. Poincare, CNRS - Universite Pierre et Marie Curie, Paris.

2 Research supported in part by NSF grant PHY92-453. 


\section{Introduction}

One of the basic objects of study in supersymmetric quantum field theories in four dimensions is the moduli space of vacua. For example, for $N=2$ supersymmetric YangMills theories, there is a Coulomb branch in the moduli space consisting of vacua in which the gauge group $G$ is broken down to a maximal torus. The Coulomb branch is of complex dimension equal to the rank $r$ of $G$.

The secret of the Coulomb branch [1] is that it parametrizes a family of $r$-dimensional abelian varieties which controls the physics on this branch. For instance, masses of the stable massive particles are given by periods of a certain differential form, and the most interesting physical phenomena are determined by singularities of the abelian variety. An abelian variety is a complex torus with a "polarization" which in the present context comes from the symplectic pairing of electric and magnetic charge.

For $G=S U(2)$, the rank $r$ is one and one is dealing with a family of genus one Riemann surfaces depending on one complex parameter. This is a simple enough situation that it has been possible to deduce the structure rather directly using some qualitative knowledge of the physics; this was done in [1,2] for the various $S U(2)$ theories with zero or negative beta function.

A similar approach for $r>1$ would be extremely cumbersome, and one naturally looks for a short-cut. To date solutions of models with $r>1$ (with an exception mentioned at the end of this introduction) have been based on assuming that one is looking for the Jacobian (or in some cases, a Prym, the part of the Jacobian odd under a $\mathbf{Z}_{2}$ symmetry) of a family of hyperelliptic curves. With some further qualitative assumptions and physics input, it is then possible to determine the desired family. This has been carried out very effectively for $S U(N)$ [3,4], $S O(2 N+1)$ [5], and $S O(2 N)$ [6], all without matter, and also for models with matter fields in the fundamental representation [0]-10].

In general, it is not clear which models can be described by such a hyperelliptic family, or indeed by any family of Riemann surfaces (as opposed to more general abelian varieties). It is therefore natural to look for other approaches. In this paper, we start with the fact that the total space of the sought-for family of abelian varieties is a complex integrable system - this assertion will be clarified in section two. Moreover, there is a natural gauge

theory construction [11-13] of integrable systems of just the right type. So we have sought to match some of these simple integrable systems with $N=2$ models.

We have found one match, which will be the subject of this paper. It is the $S U(n)$ theory with a matter hypermultiplet in the adjoint representation; one can also think of 
this as the $N=4 S U(n)$ theory with a bare mass term for some fields breaking $N=4$ explicitly to $N=2$.

$N=4$ super Yang-Mills theory was the original example that emerged as a natural case for Montonen-Olive electric-magnetic duality [14]. This theory has zero beta function, so microscopically it has a well-defined gauge coupling constant $e$. There is no anomalous $U(1)$ global symmetry, so the physics also depends on a vacuum angle $\theta$. Montonen-Olive duality, originally formulated as an inversion of the coupling constant, is extended when the theta angle is included to an $S L(2, \mathbf{Z})$ symmetry acting on

$$
\tau=\frac{\theta}{2 \pi}+\frac{4 \pi i}{e^{2}}
$$

This generalization of the Montonen-Olive conjecture is called $S$-duality. The value of $\tau$ modulo the action of $S L(2, \mathbf{Z})$ precisely determines the isomorphism class of an elliptic curve (genus one Riemann surface) $E$. E can be described very explicitly by an equation

$$
y^{2}=\left(x-e_{1}\right)\left(x-e_{2}\right)\left(x-e_{3}\right)
$$

We will build $S$-duality into our proposed solution by including $E$ as part of the structure. In addition to $x$ and $y$, we will have a third variable $t$ and another equation

$$
F(t, x, y)=0
$$

with $F$ a rather special polynomial of degree $n$. The equations (1.2) and (1.3) for variables $x, y$, and $t$ describe a complex Riemann surface $C$ of genus $n$. The solution of the model is determined by the Jacobian of $C$ if the gauge group is $U(n)$; for $S U(n)$ one considers the Jacobian modulo the part that comes from $E$.

The precise polynomials $F$ that appear here would probably be rather hard to guess without the motivation from the integrable system; thus, our approach differs from previous investigations in that it is based on guessing a simple integrable system which then determines the more complicated equations of a Riemann surface, rather than on guessing simple equations. We hope that our approach will be useful for other examples, but some ingredients are clearly still missing to give a general recipe for solving any $N=2$ model with any gauge group and hypermultiplet representation.

As for the results that come from our solution of the model, the most important point is certainly that $S$-duality is valid for all $n$; previous computations focussed on $n=2$. There is an interesting detail in how $S$-duality is realized: the $S$-duality group turns out 
to be $S L(2, \mathbf{Z})$ rather than $P S L(2, \mathbf{Z})$; the central element -1 of $S L(2, \mathbf{Z})$ acts by charge conjugation.

Also, the model has the beautiful property that (under a small perturbation to $N=1$, as in [1]) each and every phase allowed by 't Hooft's classification of the massive phases of $S U(n)$ gauge theory [16] occurs precisely once. For general $n$, such phases are classified by subgroups of $\mathbf{Z}_{n} \times \mathbf{Z}_{n}$ of order $n$, and the number of possible phases is the sum of the positive divisors of $n$. It emerges from our solution that $S L(2, \mathbf{Z})$ acts on the phases according to its natural action on $\mathbf{Z}_{n} \times \mathbf{Z}_{n}$, as one would expect by combining the ideas of 't Hooft with $S$-duality; for $n=2$ this has been seen in [2,15]. $(S L(2, \mathbf{Z})$ action on analogous $\mathbf{Z}_{n}$ phases was first seen in a lattice model [17]

Another interesting feature of the model is that it exhibits singularities of a more general type than seen in previous soluble models. The simplest singularities of Riemann surfaces are the nodes or ordinary double points, which, as explained in [1], lead to monopole condensation and confinement. In some ways, the next simplest singularity is the cusp $y^{2}=x^{3}$ which was argued by Argyres and Douglas [18 to lead to a novel kind of superconformal critical point in four dimensions. These cusps show up also in our model, and for gauge group $S U(3)$ we determine how they are transformed by $S L(2, \mathbf{Z})$.

Other singularities of curves will lead to more general critical points. For instance, the singularity $y^{2}=x^{n}$, which can readily arise in a family of hyperelliptic curves, was also briefly discussed in [18]. Because the Riemann surface $C$ in our construction is not hyperelliptic, still more general singularities can arise. It is very plausible that for sufficiently large $n$ we can obtain an arbitrary singularity $F(x, y)=0$ of a plane curve.

This paper is organized as follows. In section two of the paper, we explain why integrable systems are relevant and describe the particular system that we believe controls the solution of the $S U(N)$ gauge theory with a matter field in the adjoint representation. The candidate for the solution of the model that arises naturally in the integrable system framework is put in an explicit form at the end of section two and then studied in section three. The paper is written in such a way that interested readers can consult section two, while those who are only interested in the solution of the model can jump to section three, much of which can be read independently of section two. In section four, we discuss a few points of physics that are needed in interpreting some of the results of section three.

Perhaps the main potential interest of our construction is the role of an auxiliary two-dimensional classical gauge theory that is used in describing the solution of the fourdimensional quantum gauge theory. This may prove to have something to do with an eventual better understanding and explanation of duality. Both the four-dimensional quantum 
gauge theory that we are solving and the two-dimensional auxiliary classical system have fields known as Higgs fields that enter the formalism in rather similar ways, though the reason for the close analogy is mysterious.

When this paper was substantially complete, there appeared a paper by Martinec and Warner [19] who used a certain class of integrable models to describe pure $N=2$ gauge theory (without hypermultiplets) with any simple gauge group. We hope that construction and the one presented here will prove to be special cases of a more general story.

\section{Curves And Integrable Systems}

\subsection{Why Integrability?}

We first wish to explain what we mean in saying that the solution of any of these $N=2$ models involves an integrable system in the complex sense.

In [1,2], $S U(2)$ gauge theories were solved in terms of a family of elliptic curves $X \rightarrow U$, where $U$ is the complex $u$ plane ( $u$ is the gauge-invariant order parameter $u=\operatorname{Tr} \phi^{2}$ ) and the fibers of the map $X \rightarrow U$ are (except at finitely many singularities) Riemann surfaces of genus one. We write the fiber corresponding to $u$ as $X_{u}$. Part of the solution of the model involves also giving a meromorphic differential one-form $\lambda$ on $X_{u}$, varying holomorphically with $u$, such that the masses of the stable particles are integer linear combinations of the fundamental periods $\vec{a}=\left(a_{D}, a\right)$ of $\lambda$. In general, $\lambda$ has poles (the theory of which can be quite elaborate; see section 17 of [2]). In addition, $\lambda$ is not uniquely determined as a one-form; a transformation $\lambda \rightarrow \lambda+d \alpha$, with $\alpha$ a meromorphic function, does not affect the periods.

There is no natural choice of $\lambda$. Rather, the "gauge-invariant" object, free of these ambiguities, is the two-form $\omega=d \lambda$, which is moreover holomorphic. Holomorphy of $\omega$ is needed, as explained in section 6 of [1], to prove positivity of the metric on $U$. Though $\omega$ does not quite determine the periods $\vec{a}$, it determines their derivative,

$$
d \vec{a}=\int_{\vec{\gamma}} \omega
$$

On the left $d$ is the exterior derivative on $U$, and on the right $\vec{\gamma}$ is a set of fundamental one-cycles on the fiber. (2.1) is just a more abstract way to write the fact that as $\vec{a}=\int_{\vec{\gamma}} \lambda$, $d \vec{a} / d u=\int_{\vec{\gamma}} d \lambda / d u$. As for the metric on $U$, it is defined as follows. One starts with the $(2,2)$ form $\omega \wedge \bar{\omega}$ on $X$. By "integrating over the fiber" of $X \rightarrow U$, one gets a $(1,1)$ form 
on $U$, which is the Kahler form of the Kahler metric on $U$. This means concretely that if locally $\omega=\alpha \wedge d u$, with $\alpha$ a holomorphic one-form on $X_{u}$, then the Kahler form on $U$ is $d \bar{u} \wedge d u f(u)$ with $f=\int_{X_{u}} \alpha \wedge \bar{\alpha}$. This is manifestly positive as long as $\alpha \neq 0$, that is, as long as $\omega \neq 0$.

Now let us consider the generalization of this to the case of a gauge group $G$ of rank $r>1$. As explained in [1], section 3, the base $U$ is now a complex manifold of dimension $r$ (a copy of $\mathbf{C}^{r}$ parametrized by the gauge invariant order parameters) and the physics is described by a family of $r$-dimensional complex tori $X_{u}$, which are fibers of a map $X \rightarrow U$, with $X$ a complex manifold of dimension $2 r$. As described in [1], the physics is then determined from a meromorphic one-form $\lambda$ whose restriction to the fibers is closed. But $\lambda$ has ambiguities and complicated poles as just explained; the natural "gauge-invariant" object is $\omega=d \lambda$ which is a closed holomorphic two-form. Concretely

$$
\omega=\sum_{i} d u^{i} \frac{d \lambda}{d u^{i}}
$$

where $u^{i}$ - the gauge-invariant order parameters - are coordinates on $U$. Because the restriction of $\lambda$ to the fibers of $X \rightarrow U$ is closed, $\omega$ is a sum of terms each proportional to at least one one-form $d u^{i}$ coming from the base; in that sense, the restriction of $\omega$ to the fibers of $X \rightarrow U$ is zero. The particle masses - or rather their derivatives with respect to $u^{i}-$ are still given by (2.1).

To define the metric, we need one further subtlety. If one ignores the complex structure, $X_{u}$ is a real torus of dimension $2 r$ whose first homology is the $2 r$-dimensional space of magnetic and electric charges in this theory (whose low energy gauge group is $U(1)^{r}$ ). Given two particles with magnetic and electric charge vectors $\left(\vec{g}_{1}, \vec{e}_{1}\right)$ and $\left(\vec{g}_{2}, \vec{e}_{2}\right)$, respectively, there is a natural symplectic pairing $\vec{g}_{1} \cdot \vec{e}_{2}-\vec{e}_{1} \cdot \vec{g}_{2}$ which according to the Dirac quantization law is integer-valued. This pairing is equivalent to a two-form $t$ on $X_{u}$ which has integral periods and moreover (because of the constraints of $N=2$ supersymmetry) is positive and of type $(1,1)$ in the complex structure on $X_{u}$. Such an object defines what is called a "polarization" of $X_{u}$. 3 Endowed with this polarization, our complex torus

3 If all electric and magnetic charges allowed by Dirac quantization actually appear in the system, the polarization will be principal; but this is not so in general. If one relaxes the positivity condition on $t$, one gets an indefinite polarization such as arises typically in intermediate Jacobians of higher dimensional varieties, cf. [20,21], where the varieties are Calabi-Yau threefolds. 
becomes an Abelian variety (which is simply a complex torus that can be described by algebraic equations).

The metric can now be defined as follows: starting with the $(r+1, r+1)$-form $t^{r-1} \wedge$ $\omega \wedge \bar{\omega}$ on $X$, integrate over the fibers of $X \rightarrow U$ to get a $(1,1)$ form on $U$ which will be the Kahler form of the Kahler metric on $U$. The metric obtained this way will always be positive semi-definite and will be positive definite if and only if $\omega$ is non-degenerate, that is if and only if in any local coordinate system on $X$, the matrix $\omega_{I J}, I, J=1 \ldots 2 r$ of components of $\omega$ is invertible. In fact, more concretely in our case non-degeneracy means that locally one can pick the coordinates to be $u^{i}, i=1 \ldots r$ and some "conjugate" variables $x_{i}, i=1 \ldots r$ along the fibers, with $\omega=\sum_{i} d x_{i} \wedge d u^{i}$. (If the restriction of $\omega$ to the fibers were non-zero, there would be additional terms $\left.d x_{i} \wedge d x_{j}.\right) \omega$ being non-degenerate is equivalent to the fact that all the $d u^{i}$ appear in this formula; if one of them were absent, the metric on $U$ would vanish in the corresponding direction and so would not be strictly positive. Thus, $\omega$ is non-degenerate at least where the physics is non-singular; for the $S U(2)$ models, $\omega$ is non-degenerate even at singular fibers, but it is not clear whether this is general.

At least away from singular fibers, the basic structure is therefore a family of abelian varieties $X \rightarrow U$ endowed with a non-degenerate, closed, holomorphic two-form $\omega$, which moreover has vanishing restriction to the fibers. If we just require $\omega$ to be non-degenerate, closed, and holomorphic, then it defines a complex symplectic structure on $X$. This enables one to define Poisson brackets of holomorphic functions much as one usually defines Poisson brackets of functions on an ordinary symplectic manifold. Thus, denoting as $\omega^{I J}$ the inverse matrix of $\omega_{I J}$, the Poisson bracket of two local holomorphic functions $f$ and $g$ is defined by the usual formula

$$
\{f, g\}=\sum_{I, J} \omega^{I J} \partial_{I} f \partial_{J} g .
$$

As in the usual case, the fact that $d \omega=0$ implies that this Poisson bracket obeys the Jacobi identity. We have not yet used the fact that the restriction of $\omega$ to the fibers vanishes. This means, as above, that $\omega$ can be written locally as $\sum_{i} d x_{i} \wedge d u^{i}$, and therefore that the Poisson brackets $\left\{u^{i}, u^{j}\right\}$ vanish, that is, the $u^{i}$ are a maximal set of commuting Hamiltonians. This is the basis for asserting that we are dealing with the complex, or even algebraic, analogue of a completely integrable Hamiltonian system. We call such an object an algebraically completely integrable Hamiltonian system. 
Conversely, given an algebraically completely integrable Hamiltonian system $X$, one can reconstruct much of this structure. Defining $U$ to be the space parametrized by the commuting Hamiltonians, one has a map $X \rightarrow U$ (which forgets the other variables). If this map is proper, one can prove that the fibers are complex tori (otherwise we could get products of tori and affine spaces.) In any case, $\omega$ has vanishing restriction to the fibers.

\section{Dependence On Mass Terms}

So far, we have described the structure that appears in a single $N=2$ supersymmetric gauge theory. Whenever hypermultiplets are present, one gets a family of such theories, depending on certain complex mass parameters $m_{\lambda}$. Then $X$ and $\omega$ vary holomorphically with the $m_{\lambda}$, subject to a condition explained in section 17 of [2]; the cohomology class [ $\omega$ ] of the two-form $\omega$ varies linearly in the $m_{\lambda}$. This condition makes sense because, though the complex structure of $X$ varies with the $m_{\lambda}$, as a real manifold (of dimension $\left.4 r\right) X$ is fixed, and in particular its real cohomology, where $[\omega]$ takes values, is locally a fixed vector space. (Globally, we are dealing with a vector bundle with flat connection. Its base, parametrizing values of the mass parameters $m_{\lambda}$ for which the corresponding $X$ is non-singular, is an open subset in a vector space, while its fibers are the cohomologies of those $X$.)

The following very natural way to get a family $X, \omega$ with $[\omega]$ varying linearly with respect to some parameters will be used in the sequel. Suppose that one is given a complex symplectic manifold $Y, \omega$ admitting the action of a complex Lie group $H$. The action of $H$ is generated by holomorphic vector fields $V_{a}, a=1 \ldots \operatorname{dim} H$. Just as in ordinary classical

mechanics, one can now look for holomorphic functions $h_{a}$ that generate the action of $V_{a}$ by Poisson brackets. (In components, this means, as usual, that $\partial_{I} h_{a}=\omega_{I J} V_{a}^{J}$.) As in mechanics, one can now "reduce" with respect to the $H$ action, the prototype being the reduction of the two-body problem with a central force to a radial problem. One does this by considering only orbits with

$$
h_{a}=\mu_{a},
$$

with the $\mu_{a}$ being some complex constants, and dividing by the subgroup $H^{\prime}$ of $H$ that leaves fixed the $\mu_{a}$. (For instance, in mechanics $H$ might be the rotation group in the two-body problem, the $h_{a}$ would be the components of angular momentum, (2.4) would assert that the angular momentum has a fixed value that points in, say, the $z$ direction, and $H^{\prime}$ would be the group of rotations around the $z$ axis.) The space of solutions of (2.4) divided by $H^{\prime}$ is a complex manifold $X$ which is endowed with a complex symplectic 
form induced from $\omega$ (which we will again refer to as $\omega$ ). The basic fact we need about this situation is that if one lets the $\mu_{a}$ vary, keeping $H^{\prime}$ fixed (in the example, with $H^{\prime}$ the group of rotations around the $z$ axis, $\mu_{a}$ would be an arbitrary vector pointing in the $z$ direction), then the symplectic structure of $\omega$ varies linearly with the $\mu_{a}$ [22]. (For real symplectic manifolds, this result enters the theory of linear sigma models in an essential way [23].) When $X$ and $Y$ are related as above, $X$ is said to be a symplectic quotient of $Y$ by $H^{\prime}$.

Therefore, this setup, with the $\mu_{a}$ playing the role of the bare masses in an $N=2$ supersymmetric gauge theory, gives a natural way to automatically obey the constraint that $[\omega]$ varies linearly in the masses. As was seen in section 17 of [2], that constraint is very powerful (completely determining, for instance, the solution of the $S U(2)$ theory with $N_{f}=4$ ) but very complicated to implement directly. A construction that obeys this constraint a priori is therefore highly desireable.

\subsection{Gauge Theory And Integrable Systems}

In what follows, we use a natural construction of algebraically integrable systems as symplectic quotients. The construction of such a system, associated to an arbitrary Riemann surface and reductive group, is due to Hitchin [11]. In order to obtain interesting families of symplectic quotients, we use a version of this construction due to Markman [12,13], which uses a marked Riemann surface with specified conjugacy classes at the marked points. Actually, we will only describe the small part of this beautiful story that we need, and thus will not attempt to describe the hyper-Kahler structure associated with these systems, or the parts of the story that are purely topological in nature, independent of complex structures.

We start with a complex Riemann surface $\Sigma$ and compact gauge group $G$. We let $A$ be a connection on a $G$-bundle over $\Sigma$ - for simplicity we take it to be the trivial bundle. And we let $\Phi$ be a one-form with values in the adjoint representation of $G$. Let $Y$ be the space of such pairs. We give $Y$ a complex structure by saying that the $(0,1)$ part of $A$ and the $(1,0)$ part of $\Phi$ are holomorphic. A complex symplectic structure is defined on $Y$ by saying that the non-zero Poisson brackets of the (holomorphic) components of $A$ and $\Phi$ are

$$
\left\{A_{z}^{a}(x), \Phi_{z}^{b}(y)\right\}=\delta^{a b} \delta(x, y) .
$$

4 Also, we will glide over various subtleties associated with unstable and semi-stable points in quotients, so various assertions are only true on dense open sets - good enough for our purposes. 
Thus all components of $\{A, A\}$ and $\{\Phi, \Phi\}$ vanish.

The group $H_{0}$ of $G$-valued gauge transformations acts on $Y$ in the usual fashion, preserving the complex structure. Slightly less obvious is the fact that the complexification $H$ of $H_{0}$, which is the group of $G_{\mathbf{C}}$-valued gauge transformations $\left(G_{\mathbf{C}}\right.$ is the complexification of $G$ ) acts holomorphically on $Y$. One simply takes $G_{\mathbf{C}}$ to act in the standard fashion on the holomorphic fields, the $(0,1)$ part of $A$ and the $(1,0)$ part of $\Phi$, while (since $A$ and $\Phi$ are real or hermitian by definition) acting in the complex conjugate fashion on the complex conjugates of these fields. Let $H$ be the group of such $G_{\mathbf{C}}$-valued gauge transformations.

Since $H$ acts holomorphically and preserving the complex structure, one can ask what are the Hamiltonian functions that generate the $H$ action under the Poisson brackets (2.5). These turn out to be the objects

$$
h=\bar{D}_{A} \phi
$$

where now $\bar{D}_{A}$ is the $\bar{\partial}$ operator determined by $A$ and $\phi$ is the $(1,0)$ part of $\Phi$. 5 Recall that the $\bar{D}_{A}$ operator gives a holomorphic structure to the trivial $G_{\mathbf{C}}$ bundle over $\Sigma$, endowing it with a complex structure. The symplectic quotient $X$ is thus the space of solutions of $h=0$, that is

$$
\bar{D}_{A} \phi=0
$$

divided by $H$.

Let us describe $X$ explicitly. First of all we forget about $\phi$. The $G_{\mathbf{C}}$ gauge transformations act on the $\bar{D}_{A}$ operator by $\bar{D}_{A} \rightarrow g \bar{D}_{A} g^{-1}$, which is the usual equivalence relation on $\bar{\partial}$ operators. So the space of $A$ 's modulo the action of $H$ is the moduli space $\mathcal{M}$ of holomorphic $G_{\mathbf{C}}$ bundles on $\Sigma$. The cotangent space to $\mathcal{M}$ is the space of holomorphic one-forms valued in the adjoint representation, that is, the space of $\phi$ 's obeying (2.7). So the symplectic quotient $X$ of $Y$ by $H$ - which should be the space of solutions of (2.7) divided by $H$ - is just the cotangent bundle $T^{*} \mathcal{M}$ of $\mathcal{M}$. 6 The symplectic structure of $X$ (obtained by restricting (2.5) to modes tangent to solutions of (2.7)) is just its natural structure as a cotangent bundle.

5 To make this very explicit, for every adjoint-valued function $\epsilon$ we have the infinitesimal gauge transformation $\delta A_{i}=-D_{i} \epsilon, \delta \Phi=[\epsilon, \Phi]$. The Hamiltonian $h(\epsilon)=\int_{\Sigma} \operatorname{Tr} \epsilon \bar{D}_{A} \phi$ generates this transformation via the Poisson brackets defined above.

6 In keeping with a previous footnote, this is actually only a valid description of the symplectic quotient on a dense open set. 
Now (following Hitchin) let us exhibit $X$ as a completely integrable system in the complex sense. To do this, if $X$ has complex dimension $2 n$, we must exhibit $n$ independent holomorphic functions that Poisson-commute, that is, $n$ commuting Hamiltonians. Suppose first that $G=S U(2)$, and let $\Sigma$ be of genus $g>1$. Then $\operatorname{dim}_{\mathbf{C}}(X)=6 g-6$, so we need $3 g-3$ commuting Hamiltonians. To find them, we simply observe that $\operatorname{Tr} \phi^{2}$ is a holomorphic function on $Y$ (since it is a function only of the $(1,0)$ part of $\Phi$ ) which moreover is gauge-invariant, so its restriction to $h=0$ descends to a holomorphic function on $X$. Moreover, $\operatorname{Tr} \phi^{2}$ is a quadratic differential on $\Sigma$ which is holomorphic when $h=0$, Holomorphy of $\operatorname{Tr} \phi^{2}$ means that it can be paired with $H^{1}(\Sigma, T)$ ( $T$ is the holomorphic tangent bundle of $\Sigma$ ) by integration. For $\alpha \in H^{1}(\Sigma, T)$, the formula

$$
v(\alpha)=\int_{\Sigma} \alpha \wedge \operatorname{Tr} \phi^{2}
$$

defines a holomorphic function on $X$. As these functions are constructed from $\phi$ only, they Poisson-commute, in view of the structure of the Poisson brackets. Since $H^{1}(\Sigma, T)$ has dimension $3 g-3$, we get the desired $3 g-3$-dimensional space of holomorphic Poissoncommuting functions.

For more general gauge groups, one repeats the above construction, using all the independent gauge-invariant polynomials in $\phi$ and not only the quadratic function $\operatorname{Tr} \phi^{2}$. For instance, for $S U(n)$, one uses the independent invariants $\operatorname{Tr} \phi^{k}$ for $k=2,3, \ldots, n$. Since $\operatorname{Tr} \phi^{k}$ is a holomorphic $k$-differential on $\Sigma$, and the space of such holomorphic $k$ differentials has dimension $(2 k-1)(g-1)$ for $k>1$, one gets the correct number $(3 g-$ $3)+(5 g-5)+\ldots+(2 n-1)(g-1)=\left(n^{2}-1\right)(g-1)$ of commuting Hamiltonians.

\section{Reduction With Respect To A Subgroup}

One can generalize this and take the symplectic quotient with respect to a finitecodimension subgroup $H^{\prime}$ of $H$. To do so, following [12], we select an arbitrary finite set of points $P_{(i)}, i=1, \ldots, d$ on $\Sigma$. At each $P_{(i)}$, pick an element $\mu_{(i)}$ of the Lie algebra of $G$. Instead of setting $h=0$, set $h$ to a sum of delta functions supported at the $P_{(i)}$. In view of the definition of $h$, one does this by imposing the equation

$$
\bar{D}_{A} \phi(x)=\sum_{i} \mu_{(i)} \delta\left(x, P_{(i)}\right) .
$$

This condition means that $\phi$ is holomorphic away from the $P_{(i)}$, and has simple poles at the $P_{(i)}$ with residue equal to $\mu_{(i)}$. 
Next we divide by $H^{\prime}$, the subgroup of $H$ that commutes with the $\mu_{(i)}$. If we ignore $\phi$, then the quotient of the space of connections by $H^{\prime}$ is simply the moduli space $\mathcal{M}_{\vec{\mu}}$ of holomorphic $G_{\mathbf{C}}$ bundles on $\Sigma$ with the structure group reduced at $P_{(i)}$ to the subgroup of $G_{\mathbf{C}}$ that commutes with $\mu_{(i)}$. Including $\phi$ and also imposing (2.9), the symplectic quotient $X_{\vec{\mu}}$ of $Y$ by $H^{\prime}$ is the space of pairs consisting of a point in $\mathcal{M}_{\vec{\mu}}$ and a solution $\phi$ of (2.9).

Complete integrability is again established by looking at the components of gaugeinvariant pluri-differentials such as $\operatorname{Tr} \phi^{2}$. If we let the $\mu_{(i)}$ vary while keeping fixed $H^{\prime}$, then - in keeping with a finite-dimensional phenomenon explained above - the cohomology class of the induced symplectic structure on $X_{\vec{\mu}}$ varies linearly.

So the general conditions are right to describe an $N=2$ supersymmetric gauge theory in four dimensions by such an integrable system, with the components of $\mu$ being linear functions of the bare masses.

For readers interested in the classical geometry in more depth, it may be helpful to compare the symplectic objects $T^{*} \mathcal{M}_{\vec{\mu}}$ and $X_{\vec{\mu}}$. Over a general curve $\Sigma$, let $\pi: \mathcal{M}_{\vec{\mu}} \longrightarrow \mathcal{M}$ be the natural projection. Its fibers are coadjoint orbits, hence are naturally symplectic. The cotangent bundle $T^{*} \mathcal{M}_{\vec{\mu}}$ has a corresponding subbundle $\pi^{*} T^{*} \mathcal{M}$, which inherits a symplectic structure from $T^{*} \mathcal{M}_{\vec{\mu}}$ and the coadjoint fibers. Our object $X_{\vec{\mu}}$ looks locally like $\pi^{*} T^{*} \mathcal{M}$, in fact it is an affine bundle over $\mathcal{M}_{\vec{\mu}}$ modelled on the vector subbundle $\pi^{*} T^{*} \mathcal{M}$ of $T^{*} \mathcal{M}_{\vec{\mu}}$. On the other hand, if $\Sigma$ is such that the general vector bundle on it has a non-trivial group $A$ of automorphisms, then the fibers of $\pi$ are coadjoint orbits modulo $A$, and $X_{\vec{\mu}}$ is now modelled on the subquotient $\pi^{*} T^{*} \mathcal{M} / A$ of $T^{*} \mathcal{M}_{\vec{\mu}}$, which is still symplectic. (In the case of interest to us, $\Sigma$ has genus 1 , so there is always a large $A$.)

\subsection{An Example}

As promised in the introduction, we will attempt to describe in this way the $N=4$ theory with gauge group $S U(n)$ perturbed by a bare mass that reduces the symmetry to $N=2$. First, though, we consider the $N=4$ theory without the mass perturbation.

We need to pick a Riemann surface; what will it be? Because the anomalies vanish, this theory has a microscopic $\tau$ parameter

$$
\tau=\frac{\theta}{2 \pi}+\frac{4 \pi i}{e^{2}}
$$

In seeking the solution, we will assume that the theory has complete $S L(2, \mathbf{Z})$ invariance. The orbit of $\tau$ modulo $S L(2, \mathbf{Z})$ precisely determines the isomorphism class of a genus 
one curve $E$. This gives the Riemann surface we need. We will proceed by constructing integrable systems via gauge theory on $E$.

We also need a gauge group for the gauge theory on $E$. We will simply use the same gauge group $G$ that entered the four-dimensional problem we are trying to solve.

Using $E$ (without marked points) and $G$ in the above construction of an integrable system, we get a definite integrable system with nothing that can be adjusted. Does it reproduce the vacuum structure of the $N=4$ theory? $\mathrm{G}$ We claim that it does (for any compact $G$, and not just the case $G=S U(N)$ to which we restrict later).

To see this, first take $G=U(1)$. The curve $E$ can be described by an explicit equation

$$
y^{2}=\left(x-e_{1}\right)\left(x-e_{2}\right)\left(x-e_{3}\right)
$$

in the $x-y$ plane; the $e_{i}$ are distinct complex numbers. For $G=U(1)$ and $E$ of genus one, the moduli space $\mathcal{M}$ of flat $G_{\mathbf{C}}$ bundles on $E$ is just a copy of $E$. $\phi$ is supposed to be a holomorphic differential on $E$ with values in the adjoint representation; because $G$ is abelian, $\phi$ is just an ordinary holomorphic differential on $E$. Any such differential is $\phi=a \phi_{0}$ with $\phi_{0}$ a fixed holomorphic differential on $E$ and $a \in \mathbf{C}$. The integrable system $X$ is therefore $E \times \mathbf{C}$, with $\mathbf{C}$ being the $a$-plane. (As a check, note that $X$ should be $T^{*}(\mathcal{M})$, but with $\mathcal{M}$ being the torus $E, T^{*} \mathcal{M}$ is the same as $E \times \mathbf{C}$.) The symplectic form on $X$ is the natural symplectic form on $T^{*}(\mathcal{M})$ :

$$
\omega=\frac{d x}{y} \wedge d a
$$

where $d x / y$ is a holomorphic differential on $E$. The Kahler form on the $a$-plane, obtained by taking $\omega \wedge \bar{\omega}$ and integrating over $E$, is

$$
\operatorname{Im}(\tau) d a \wedge d \bar{a}
$$

This flat metric is the correct free field theory metric on the $a$ plane in the $N=4 U(1)$ theory.

Now we want to generalize this for non-abelian compact $G$ of rank $r$. What is special about $N=4$ is that the correct answer for any $G$ is in fact very similar to (2.13), except

7 When we say "vacuum structure" here, we are interested in those vacua that go over to the $N=2$ Coulomb branch when the mass perturbation is added; we will ignore the expectation values of fields that are part of the $N=2$ hypermultiplet. 
that one must divide by the Weyl group. This holds because for $N=4$, the metric on the moduli space of vacua is completely determined by the symmetries, and in particular is given exactly by the tree level expression. Recall that $N=2$ relates the gauge field to a complex scalar field $\phi$ in the adjoint representation. The vacuum is determined by the value of $\phi$ up to conjugation by $G_{\mathbf{C}}$; this means that a generic $\phi$ can be diagonalized, and replaced by an $r$-tuple of complex fields $\vec{a}=\left(a_{1}, \ldots, a_{r}\right)$ taking values in a maximal abelian subalgebra of the Lie algebra, except that one must identify two $\vec{a}$ 's that differ by action of the Weyl group. The Kahler form - read off from the classical Lagrangian because there are no quantum corrections - is the obvious generalization of (2.13), namely

$$
\operatorname{Im}(\tau) \operatorname{Tr} d \vec{a} \wedge d \overline{\vec{a}}
$$

Dividing by the Weyl group $W$ of $G$, this is to be understood as a metric not on $\mathbf{C}^{r}$ but on the moduli space $U=\mathbf{C}^{r} / W$.

Let us try to reproduce this answer via gauge theory on $E$. Because $\pi_{1}(E)$ is abelian, the moduli space of semistable holomorphic $G_{\mathbf{C}}$ bundles on $E$ would be unchanged if one replaces $G$ by a maximal torus $T$, except that one has to divide by the Weyl group. Since $T=U(1)^{r}$, the moduli space of holomorphic $G_{\mathbf{C}}$ bundles is $E^{r} / W$, the product of $r$ copies of $E$ divided by $W$. The $s^{\text {th }}$ copy of $E$ is described by an equation

$$
y_{s}^{2}=\prod_{i=1}^{3}\left(x_{s}-e_{i}\right)
$$

in the $x_{s}, y_{s}$ plane. To this we must adjoin a holomorphic differential $\phi$ with values in the adjoint representation. At a generic point in $E^{r}$, holomorphy forces the part of $\phi$ that does not commute with $T$ to be zero and the remaining part to be constant, so we get a complex field $\phi=\vec{a}=\left(a_{1}, \ldots, a_{r}\right)$ with values in the abelian subalgebra, just as in the four-dimensional discussion in the last paragraph. The integrable system is thus $X=\left(E^{r} \times \mathbf{C}^{r}\right) / W$, with $\mathbf{C}^{r}$ being the product of the complex $a_{s}$-planes, $s=1, \ldots, r$; away from the fixed points of $W$, this is the same as $X=T^{*}\left(E^{r} / W\right)$. The symplectic form on $X$ (obtained by restricting the microscopic Poisson brackets to solutions of (2.7)) is

$$
\sum_{s=1}^{r} \frac{d x_{s}}{y_{s}} \wedge d a_{s} .
$$


Computing from this the metric (by integrating $t^{r-1} \wedge \omega \wedge \bar{\omega}$ over the fibers 8 ), we arrive at the desired metric (2.14). Just as in the four-dimensional field theory, we interpret this as a metric on $U=\mathbf{C}^{r} / W$, not on $\mathbf{C}^{r}$, because $X$ is really $\left(E^{r} \times \mathbf{C}^{r}\right) / W$, not $E^{r} \times \mathbf{C}^{r}$.

\section{The Mass Perturbation}

Now we want to consider the mass perturbation breaking $N=4$ to $N=2$. This perturbation depends on precisely one complex parameter $m$, and so we need a generalization of the above to a family of complex integrable systems with cohomology class varying linearly with $m$. To do so, as motivated in the last subsection, we will let $\phi$ have poles, with residue linear in $m$. We must pick the allowed position and conjugacy classes of the poles.

There are two strong constraints. First, we want to maintain the full $S L(2, \mathbf{Z})$ invariance of the above construction. For this, we can single out a single point $P$ at which a pole will be allowed, but singling out more than one point would break part of the $S L(2, \mathbf{Z})$ symmetry. For the genus one curve

$$
y^{2}=\prod_{i=1}^{3}\left(x-e_{i}\right)
$$

we will take $P$ to be the point at $x=y=\infty$. A genus one curve with one point selected is called an elliptic curve.

What remains is to identify the $\mu$, that is, the conjugacy class of the pole in $\phi$ at $P$. Here we run into our second strong constraint. The integrable system $X$ just introduced has the correct dimension - we saw above how the components of $\phi$ naturally turn into the vacuum parameters on the Coulomb branch. For generic $\mu$, the integrable system $X_{\mu}$ has a dimension bigger than that of $X$. For example, for $G=S U(n)$, the case we consider in the rest of this paper, there is a unique conjugacy class of $\mu$ (up to scaling) that leads to $X_{\mu}$ of the correct dimension. This is the case, considered in [24] in the context of elliptic solitons, in which $\mu$ is a diagonalizable matrix whose eigenvalues are (a constant multiple of) $1,1, \ldots, 1,-(n-1)$; that is, $n-1$ eigenvalues equal 1 , and one equals $-(n-1)$. We therefore have precisely one candidate for the solution of the $N=4 S U(n)$ theory with the mass term; the rest of this paper is devoted to giving evidence that it is correct.

8 An elliptic curve $E$ has a natural polarization $t_{0}$ - the cohomology class dual to a point. The polarization $t$ of $E^{r}$ that comes from the two-dimensional gauge theory construction is the sum of the polarizations on the factors. 
The reason that allowing a pole of just this type does not increase the dimension of $X$ is the following. Recall that $X_{\mu}$ is modelled on a subquotient $\pi^{*} T^{*} \mathcal{M} / A$ of $T^{*} \mathcal{M}_{\mu}$, where $A$ is the group of automorphisms of a general vector bundle $\left(=\left(\mathbf{C}^{*}\right)^{n-1}\right.$, in our case), and

$\pi: \mathcal{M}_{\mu} \longrightarrow \mathcal{M}$ is the natural projection. In our case, the dimension of $A$ equals the fiber dimension of $\pi$ (equals $n-1$ ), so there is no change in the dimension of $X$. The key fact is that the dimension of the coadjoint orbit of $\mu$ is twice the dimension of $A$.

\subsection{Spectral Curves}

The discussion may sound abstract, and one might despair of being able to calculate. What makes this possible is one more ingredient in Hitchin's story, beyond what we have so far explained; this is the notion of a spectral cover. In explaining this, we will take $G=S U(n)$, so $G_{\mathbf{C}}=S L(n, \mathbf{C})$.

A point in the integrable system $X$ is a holomorphic $G_{\mathbf{C}}$ bundle $V$ together with an adjoint-valued holomorphic one-form $\phi$ on the Riemann surface $\Sigma$ of genus $g$. Consider the $n$-sheeted cover of the genus- $g$ surface $\Sigma$ given by the equation

$$
\operatorname{det}(t-\phi)=0
$$

where $t$ takes values in the canonical line bundle of $\Sigma$. This gives a Riemann surface $C$, of genus $\widetilde{g}:=\left(n^{2}-1\right)(g-1)+g$. The claim is that everything can be described from the structure of $C$. This makes it possible to reduce the story from a general discussion of families of abelian varieties to very concrete issues about Riemann surfaces given by concrete equations.

Note first of all that there is no problem in making concrete the equation (2.18). This equation can be written out very explicitly

$$
t^{n}+t^{n-2} W_{2}(\phi)+t^{n-3} W_{3}(\phi)+\ldots+W_{n}(\phi)=0 .
$$

The $W_{k}(\phi)$ are concrete gauge-invariant polynomials in $\phi$, and are, in fact, holomorphic $k$-differentials on $\Sigma$ (possibly with poles of a specified kind). If one expands the $W_{k}$ in a basis of $k$-differentials, the coefficients that arise are just the values of the commuting Hamiltonians of the integrable system.

What remains is to understand the fibers of the map $X \rightarrow U$ from the integrable system $X$ to the space $U$ of commuting Hamiltonians. Over a generic point $w \in \Sigma$, the equation (2.18) has $n$ distinct solutions for $t$, corresponding to one-dimensional eigenspaces 
of $\phi$. Let $v$ be a general point in $C$. It sits over some point $w \in \Sigma$, and corresponds to a one-dimensional eigenspace of $\phi(w)$. Call this eigenspace $L_{v} . L_{v}$ varies holomorphically with $v$, as the fibers of a holomorphic line bundle $\mathcal{L} \rightarrow C$.

From $\mathcal{L}$, the $S L(n, \mathbf{C})$ bundle $V \rightarrow \Sigma$ can be reconstructed as follows: if the distinct points lying over $w \in \Sigma$ are $v_{i}(w)$, then the fiber of $V$ over $w$ is

$$
V_{w}=\oplus_{i=1}^{n} L_{v_{i}(w)}
$$

This assertion is just the reconstruction of $V_{w}$ as the sum of the eigenspaces of $\phi$. If $V$ were simply a $G L(n, \mathbf{C}$ ) bundle (as would be the case for gauge group $G=U(n)$ ) we would stop here: every $V \rightarrow \Sigma$ gives a line bundle $\mathcal{L} \rightarrow C$, and conversely by (2.20), so the fiber of the map $X \rightarrow U$ would be the Jacobian of $C$.

For gauge group $G=S U(n)$, we want $V$ to be an $S L(n, \mathbf{C})$ bundle, which means that the determinant line bundle $\operatorname{det}(V)$ of $V$ should be trivial. Given a line bundle $\mathcal{L}$ on $C$, we can define a holomorphic line bundle $N(\mathcal{L})$ on $\Sigma$ whose fiber at $w \in \Sigma$ is

$$
N(\mathcal{L})_{w}=\otimes_{i=1}^{n} L_{v_{i}(w)}
$$

If $V$ is as in (2.20), then $\operatorname{det}(V)=N(\mathcal{L}) \otimes K_{\Sigma}{ }^{\otimes n(n-1) / 2}$, cf. [25]; hence the condition that $V$ is an $S L(n, \mathbf{C})$ bundle is that $N(\mathcal{L})$ should be the appropriate multiple of $K_{\Sigma}$. So the fiber of the map $X \rightarrow U$ is, up to shifts, the kernel of the map $\mathcal{L} \rightarrow N(\mathcal{L})$ from the Jacobian of $C$ to that of $\Sigma$. In the case that $\Sigma$ is elliptic, there will be no shift, as $K_{\Sigma}$ is trivial.

\section{Detailed Recipe}

For the case we actually want to look at, $\Sigma$ is the genus one curve defined by

$$
y^{2}=\prod_{i=1}^{3}\left(x-e_{i}\right)
$$

for some complex numbers $e_{i}$, and $\phi$ is permitted to have a simple pole at the point $P$ with $x=y=\infty$. The residue of $\phi$ is to be a matrix with eigenvalues a constant times $1,1, \ldots, 1,-(n-1)$. The constant is a multiple of the bare mass of the hypermultiplet. Because the $N=4$ theory is scale-invariant, the actual value of the bare mass (given that it is non-zero) and thus of the constant in the residues does not matter. 9

9 To be more precise, the absolute value of $m$ can be removed by scaling, and the phase by one of the $S U(4)$ global symmetries of the $N=4$ theory. 
To work near $P$, we set $x=u^{-2}$ and $y=v u^{-3}$, where $u$ is a local parameter near $P$ and $v$ is holomorphic at $u=0$. We fix the normalization of the poles in $\phi$ by requiring that near $u=0$, the polar part of $\phi$ is precisely $1 / u$ times a matrix of eigenvalues $1,1, \ldots, 1,-(n-1)$. The spectral cover $C$ of $E$ is given by an equation $\operatorname{det}(t-\phi)=0$. Setting $F(t, x, y)=$ $\operatorname{det}(t-\phi)$, we have

$$
F(t, x, y)=t^{n}+B_{2}(x, y) t^{n-2}+\ldots+B_{n}(x, y)
$$

where the $B_{k}$ are polynomials in $x$ and $y$ (so as to have no singularities except at $P$ ) and grow at most as $u^{-k}$ for $u \rightarrow 0$. Moreover, if we regard the equation $F(t, x, y)=0$ as an equation for $t$ with $x$ and $y$ fixed, then of the $n$ roots, $n-1$ have $t$ growing as $1 \cdot u^{-1}$ near $u=0$, and one root behaves as $-(n-1) u^{-1}$. This is because the roots of $\operatorname{det}(t-\phi)=0$ are just the eigenvalues of $\phi$, and their growth for $u \rightarrow 0$ is determined by the polar part of $\phi$.

The condition on behavior of the roots means that if we set $t^{\prime}=t-u^{-1}$, then only one solution for $t^{\prime}$ has a pole at $u=0$. That means that near $u=0$

$$
F\left(t^{\prime}, u\right)=F_{0}\left(t^{\prime}, u\right)+\frac{1}{u} F_{1}\left(t^{\prime}, u\right)
$$

with $F_{0}$ and $F_{1}$ holomorphic at $u=0$. As we will see at the beginning of section three, this information, together with the facts mentioned in the last paragraph, completely determines $F$ - and therefore the spectral curve $C$ - in terms of the expected parameters. For now, though, we interrupt the derivation so that readers who have chosen to omit the present section can rejoin us in section three.

\section{Properties Of The Solution}

Our proposal for solving the four-dimensional $S U(n)$ gauge theory with a massive hypermultiplet in the adjoint representation (we will set the bare mass to one and not mention it explicitly) involves a Riemann surface $C$ described by writing two equations for three variables $x, y$, and $t$. One is the equation

$$
y^{2}=\left(x-e_{1}\right)\left(x-e_{2}\right)\left(x-e_{3}\right)
$$

for a genus one Riemann surface $E$ whose $\tau$ parameter should equal $\theta / 2 \pi+4 \pi i / e^{2}$, with $e$ and $\theta$ the coupling constant and theta angle of the theory that we wish to solve. 
The second equation is

$$
F(t, x, y)=0
$$

where $F$ will be described presently. Together with (3.1), $F$ describes a curve $C$ of genus $n$. The physics is described by the part of the Jacobian of $C$ that does not come from $E$.

The first condition on $F$ is that $F$ is a polynomial in $t, x$, and $y$ which - if we consider $x, y$, and $t$ to be of degree 2,3 , and 1 respectively - contains only terms of degree $\leq n$. We also may as well assume (since we are imposing also (3.1)) that $F$ contains only terms at most linear in $y$. Moreover, in its $t$-dependence, $F=t^{n}+O\left(t^{n-2}\right)$. These conditions would leave many free parameters in $F$. To fix them, we need a further condition on the behavior of $F$ for $x, y \rightarrow \infty$. Near infinity, write $x=u^{-2}, y=u^{-3} v$, with

$$
v^{2}=\prod_{i=1}^{3}\left(1-e_{i} u^{2}\right) .
$$

Thus, in particular, $v$ is holomorphic near $u=0$ and has value $v=1$ there; $u$ is a good local parameter near $x=y=\infty$. The last condition is that if $F$ is written in terms of $u$ and $t^{\prime}=t-u^{-1}$, then $F\left(t^{\prime}, u\right)$ has only a first order pole at $u=0$, that is,

$$
F=F_{0}\left(t^{\prime}, u\right)+u^{-1} F_{1}\left(t^{\prime}, u\right)
$$

where $F_{0}$ and $F_{1}$ are holomorphic at $u=0$. These conditions together determine $F$ in terms of $n-1$ complex parameters; these are the expected parameters on the Coulomb branch.

In fact, it turns out that if we further required that $F(t, 0,0)=t^{n}$, there would be a unique polynomial $F$ obeying the constraints of the previous paragraph. There is one such polynomial for each $n$; let us call it $P_{n}$. Then the general $F$ allowed by the conditions of the previous paragraph is

$$
F=P_{n}+A_{2} P_{n-2}+A_{3} P_{n-3}+\ldots+A_{n} P_{0} .
$$

Here the $A_{i}$ are complex constants which we want to identify with the order parameters on the Coulomb branch. That is, the $A_{i}$ are gauge invariant polynomials in the scalar field $\phi$ that is related to the $S U(n)$ gauge field by $N=2$ supersymmetry. In the massless theory as analyzed in section two, the $A_{i}$ are the $i^{t h}$ elementary symmetric functions of $\phi$ (up to multiplicative constants that depend on how $\phi$ is normalized); depending on the precise formalism, there may be mass-dependent corrections to this. (In the formalism of section 
2, there is another object defined on the Riemann surface $E$, curiously also often called a Higgs field, whose characteristic polynomial also involves the $A_{i}$. The relation between the two needs a better explanation.)

Let us work out explicitly the first few $P_{k}$. For $P_{0}$ and $P_{1}$, it is impossible to have any terms at all involving $x$ and $y$, since they have degree $\geq 2$, so

$$
\begin{aligned}
& P_{0}=1 \\
& P_{1}=t .
\end{aligned}
$$

For $P_{2}$, the general polynomial obeying all conditions except the behavior at $u=0$ is $t^{2}+\alpha x$ with $\alpha$ a constant. Imposing the condition that $P_{2}\left(t^{\prime}, u\right)$ has only a simple pole at $u=0$, we get

$$
P_{2}=t^{2}-x
$$

Similarly, starting with $P_{3}=t^{3}+\alpha x t+\beta y+\gamma x$, we get

$$
P_{3}=t^{3}-3 x t+2 y \text {. }
$$

To go farther, it is useful to note that the conditions by which the $P_{n}$ are determined imply that $d P_{n} / d t=n P_{n-1}$, so that once $P_{n-1}$ is known, one knows $P_{n}$ modulo a polynomial in $x$ and $y$ only. With a little more work one gets

$$
\begin{aligned}
& P_{4}=t^{4}-6 x t^{2}+8 y t-3 x^{2}+4 x \sum_{i} e_{i} \\
& P_{5}=t^{5}-10 x t^{3}+20 y t^{2}+\left(-15 x^{2}+20 x \sum_{i} e_{i}\right) t+4 x y-8 y \sum_{i} e_{i}
\end{aligned}
$$

and so on.

Though these formulas will suffice for our applications, the general form of the $P_{n}$ 's can be described more systematically as follows. Write

$$
P_{n}(t)=\sum_{i}\left(\begin{array}{c}
n \\
i
\end{array}\right) f_{i, n}(u) t^{n-i}
$$

The condition $n P_{n-1}=P_{n}^{\prime}$ gives $f_{i, n}=f_{i, n-1}=: f_{i}$, independent of $\mathrm{n}$. Our main condition is that, substituting $t=t^{\prime}+1 / u, P_{n}$ should have pole order $\leq 1$ in $u$. In particular, setting 
$t^{\prime}=0$, i.e. $t=1 / u$, we find that the following expression can have at worst first order poles in $u$ :

$$
\begin{aligned}
\sum_{n} \frac{1}{n !} P_{n}\left(\frac{1}{u}\right) s^{n}= & \sum_{n, i} \frac{1}{i !(n-i) !} \frac{f_{i}(u)}{u^{n-i}} s^{n}=\sum_{i, j} \frac{1}{i ! j !} \frac{f_{i}(u)}{u^{j}} s^{i+j} \\
& =\left(\sum_{i} \frac{f_{i}(u)}{i !} s^{i}\right)\left(\sum_{j} \frac{(s / u)^{j}}{j !}\right)=e^{s / u} F(u, s),
\end{aligned}
$$

where $F(u, s):=\sum_{i} \frac{f_{i}(u)}{i !} s^{i}$ is a holomorphic function $F:(E \backslash \infty) \times \mathbf{C} \rightarrow \mathbf{C}$ satisfying (and uniquely characterized by): (i) $F(s u, s)$ is holomorphic in $s$ (i.e. $\left(\frac{\partial}{\partial s}\right)^{i} F(u, 0)$ has pole order $\leq i$ in $u$ ); (ii) $e^{s / u} F(u, s)$ has pole order $\leq 1$ in $u$. These conditions should translate into an explicit formula for the generating function $F$ in terms of theta functions and exponential terms on $E$.

\section{S-Duality}

It is now straightforward to exhibit the $S$-duality of the formalism. According to classical formulas used in section 16 of [2], introduce the theta functions

$$
\begin{aligned}
& \theta_{1}(\tau)=\sum_{n \in \mathbf{Z}} q^{\frac{1}{2}(n+1)^{2}} \\
& \theta_{2}(\tau)=\sum_{n \in \mathbf{Z}}(-1)^{n} q^{\frac{1}{2} n^{2}} \\
& \theta_{3}(\tau)=\sum_{n \in \mathbf{Z}} q^{\frac{1}{2} n^{2}}
\end{aligned}
$$

with $q=e^{2 \pi i \tau}$, and set

$$
\begin{aligned}
& e_{3}-e_{2}=\theta_{1}^{4}(\tau) \\
& e_{1}-e_{3}=\theta_{2}^{4}(\tau) . \\
& e_{1}-e_{2}=\theta_{3}^{4}(\tau)
\end{aligned}
$$

With this choice, (3.1) describes an elliptic curve whose $\tau$ parameter is in fact $\tau$, and the coefficients in (3.1) transform as modular forms of $S L(2, \mathbf{Z})$ (one has $4 \prod_{i}\left(x-e_{i}\right)=$ $4 x^{3}-g_{2} x-g_{3}$ where $g_{2}$ and $g_{3}$ are Eisenstein series defining modular forms of weight four and six for $S L(2, \mathbf{Z})$ ). (3.1) is therefore invariant under the action of $S L(2, \mathbf{Z})$ on $\tau$ if $x$ and $y$ are taken to transform as modular forms of weight two and three, respectively.

The modular covariance extends to our second equation $F(t, x, y)=0$, if we take $t$ to transform as a modular form of weight one, and $A_{k}$ to transform as a modular form 
of weight $k$. This result about the modular weight of the $A_{k}$ extends the result in [2] for $n=2$, where $u=A_{2}$ was found to transform with weight two.

Having $A_{k}$ transform with weight $k$ means that under $\tau \rightarrow(a \tau+b) /(c \tau+d)$,

$$
A_{k} \rightarrow \frac{A_{k}}{(c \tau+d)^{k}}
$$

In particular, under the element -1 of the center of $S L(2, \mathbf{Z})$, one has

$$
A_{k} \rightarrow(-1)^{k} A_{k}
$$

This operation is usually called charge conjugation. We have learned, then, that the $S$ duality group is really $S L(2, \mathbf{Z})$ rather than $P S L(2, \mathbf{Z})$, with the center acting by charge conjugation. For $n=2$, the center acts trivially on the gauge-invariant order parameters, though in a sense one still sees the charge conjugation by the action of $S L(2, \mathbf{Z})$ on $y$.

\subsection{Flow To The Pure $N=2$ Theory}

In the rest of this section, we extract other key properties of the solution and compare to what is known about the model independently. The first issue that we will consider is the flow to the pure $N=2$ theory. In other words, in a limit in which the bare mass goes to infinity, and $\tau$ goes to infinity as the logarithm of the mass, and one also performs suitable renormalizations of the $A_{k}$, the $N=2$ theory with the matter hypermultiplet should reduce to the pure $N=2$ theory with gauge group $S U(n)$, whose structure is already known. Let us verify this. Because of scale invariance, instead of taking the bare mass to infinity, we can keep the bare mass at one and take the $A_{k}$ to "zero"; we put the word "zero" in quotes because some additive renormalization may be involved.

We have found that the desired flow can be exhibited very simply if one takes the equation for $E$ to be

$$
y^{2}=x(x-1)(x-\lambda),
$$

with some complex $\lambda$; any $E$ can be put in this form by an affine transformation $x \rightarrow a x+b$ mapping $e_{1}, e_{2}, e_{3}$ to $0,1, \lambda$. Note that the affine transformation from (3.12) to (3.15) leaves invariant the conditions ( characterized the allowed F's. However, it does not leave invariant the further condition $P_{k}(t, 0,0)=t^{k}$ that was used to define the $P_{k}$ 's. Instead, $x \rightarrow a x+b$ will add to $P_{k}$ a linear combination of $P_{r}$ 's for $r<k$, and therefore likewise will add to $A_{k}$ a linear 
combination of $A_{r}$ for $r<k$. This generalizes the situation found in section 16 of [2] where the definition of $\operatorname{Tr} \phi^{2}$ needed to exhibit $S L(2, \mathbf{Z})$ invariance differed by an additive renormalization from the definition that was natural to exhibit the flow to the pure $N=2$ theory. (As $A_{2}=\operatorname{Tr} \phi^{2}$ and $A_{0}=1$, an additive renormalization of $\operatorname{Tr} \phi^{2}$ is a special case of a transformation adding to $A_{k}$ a linear combination of $A_{r}$ for $r<k$.) We will denote the new $A_{k}$ 's which arise if (3.5) is used together with (3.15) as $A_{k}^{\prime}$.

We can take the weak coupling limit to be $\lambda \rightarrow 0$. In that limit, we take $x=\lambda \widetilde{x}$, $y=\lambda \widetilde{y}$, where $\widetilde{x}$ and $\widetilde{y}$ are to have limits as $\lambda \rightarrow 0$. The equation for $E$ thus reduces to

$$
\widetilde{y}^{2}=-\left(\widetilde{x}-\frac{1}{2}\right)^{2}+\frac{1}{4}
$$

We now have to look at the second equation $F(t, x, y)=0$. Though a general algebrogeometric argument exhibiting the desired limit for all $n$ is possible, we will here show how it works explicitly for small $n$. Let us recall that the solution of the pure $N=2$ theory for $S U(n)$ can be described [3, 3 by the curve

$$
w^{2}=\left(z^{n}+b_{2} z^{n-2}+b_{3} z^{n-3}+\ldots+b_{n}\right)^{2}+1
$$

in the $w-z$ plane, with the $b_{k}$ being the order parameters.

For $n=2$, that is for $S U(2)$, our equation $F=0$ is

$$
t^{2}-x+A_{2}^{\prime}=0
$$

We set $t=\lambda^{1 / 2} \widetilde{t}, A_{2}^{\prime}=\lambda a_{2}$, so that this reduces to $\widetilde{t}^{2}-\widetilde{x}+a_{2}=0$. Solving for $x$ and inserting in (3.16), we get

$$
\widetilde{y}^{2}=-\left(\widetilde{t}^{2}-\frac{1}{2}+a_{2}\right)^{2}+\frac{1}{4}
$$

which with obvious substitutions (including a shift of $a_{2}$ by $1 / 2$ ) is equivalent to (3.17) for $n=2$.

For $n=3$, the equation $F=0$ reads

$$
t^{3}-3 x t+2 y+A_{2}^{\prime} t+A_{3}^{\prime}=0
$$

Now we take $t=\lambda^{1 / 3} \widetilde{t}, A_{k}^{\prime}=\lambda^{k / 3} a_{k}$. The limit of (3.20) is then

$$
\widetilde{t}^{3}+a_{2} \widetilde{t}+a_{3}+2 \widetilde{y}=0
$$


Solving for $\widetilde{y}$ and substituting in (3.16), the limit as $\lambda \rightarrow 0$ is

$$
\left(\widetilde{t}^{3}+a_{2} \widetilde{t}+a_{3}\right)^{2}=-4\left(\widetilde{x}-\frac{1}{2}\right)^{2}+1
$$

which again is equivalent to (3.17) with obvious substitutions.

The general pattern continues like this for larger $n$. One scales $t=\lambda^{1 / n} \widetilde{t}, A_{k}^{\prime}=\lambda^{k / n} a_{k}$, and one eliminates $\widetilde{x}$ or $\widetilde{y}$ depending on whether $n$ is even or odd. In the limit $\lambda \rightarrow 0$ one gets (3.17) after obvious substitutions. Note that the relation between $\tau$ and $\lambda$ for small $\lambda$ is $\lambda=q^{1 / 2}=e^{\pi i \tau}$, so our scaling involves $A_{k}^{\prime}=q^{k / 2 n} a_{k}$. In fact, according to the renormalization group, the mass scale $\Lambda$ of the pure $N=2$ theory is related to the bare mass $m$ by $\Lambda^{2 n}=q m^{2 n}$ so (as we have set $m=1$ ) our relation is $A_{k}^{\prime}=\Lambda^{k} a_{k}$, as expected; in other words, to flow to the pure $N=2$ theory, we must take $q \rightarrow 0$ while keeping the $A_{k}^{\prime}$ (whose dimension is $k$ ) fixed in units of $\Lambda^{k}$.

\subsection{Exact Description For $n=2$}

Another important check of our solution is to compare it to the results already known for $S U(2)$, that is for $n=2$.

For $n=2$, the curve $C$ is described by the equations

$$
\begin{aligned}
y^{2} & =\left(x-e_{1}\right)\left(x-e_{2}\right)\left(x-e_{3}\right) \\
0 & =t^{2}-x+A_{2} .
\end{aligned}
$$

$C$ has a $\mathbf{Z}_{2} \times \mathbf{Z}_{2}$ symmetry, with the first $\mathbf{Z}_{2}$ generated by $\alpha: y \rightarrow-y$ and the second generated by $\beta: t \rightarrow-t$.

$C$ is a curve of genus two, so its Jacobian is two-dimensional. We need to split off from the Jacobian of $C$ a one-dimensional piece that will be used to describe the model. The general recipe was explained at the end of section two, but in the present case a simplification is possible: we want the part of the Jacobian of $C$ that is invariant under the diagonal transformation $\alpha \beta$, which changes the sign of both $y$ and $t$. (This kills the period that comes from $E$ - which is associated with the differential form $d x / y$ that is odd in $y$ and even in $t$ - and leaves the other period.)

This piece of the Jacobian of $C$ is just the Jacobian of the curve obtained by considering only the $\alpha \beta$-invariant functions of $x, y$, and $t$, subject to the equations (3.23). For the basic $\alpha \beta$-invariant functions, we can pick $x, z=t^{2}$, and $w=y t$. After using the second equation in (3.23) to eliminate $z$, the first becomes

$$
w^{2}=\left(x-A_{2}\right)\left(x-e_{1}\right)\left(x-e_{2}\right)\left(x-e_{3}\right) .
$$


According to our proposal for the $S U(n)$ theory, the $S U(2)$ theory should be described by the Jacobian of this curve $D$.

Instead, in [2], the $S U(2)$ theory was described by

$$
y^{2}=\left(x-e_{1} u-e_{1}^{2}\right)\left(x-e_{2} u-e_{2}^{2}\right)\left(x-e_{3} u-e_{3}^{2}\right)
$$

A small computation shows that the cross-ratio of the four points $A_{2}, e_{1}, e_{2}, e_{3}$ agrees with that of the four points $\infty, e_{1} u+e_{1}^{2}, e_{2} u+e_{2}^{2}, e_{3} u+e_{3}^{2}$ provided $A_{2}=u+e_{1}+e_{2}+e_{3}$, so that (3.24) can be mapped to (3.25) by an $S L(2, \mathbf{C})$ transformation of $x$ given that relation between $A_{2}$ and $u$. In the manifestly $S$-dual formalism of (3.12), $e_{1}+e_{2}+e_{3}=0$ and $A_{2}=u$; more generally the two descriptions in (3.24), (3.25) differ by an additive renormalization of $u=\operatorname{Tr} \phi^{2}$.

\section{Elimination Of $y$ For $n>2$}

For $n=2$, we were able to eliminate one variable (namely $x$ ) and exhibit $C$ as a curve in the $y-t$ plane. Is there any analog of this for $n>2$ ? For $n=3$, the equation $F(t, x, y)=0$ is explicitly

$$
t^{3}-3 x t+2 y+A_{2} t+A_{3}=0
$$

One can solve this for $y$, and then substitute in the equation for $E$. One learns that the curve $C$ of the $S U(3)$ theory is the curve

$$
\frac{1}{4}\left(t^{3}-3 x t+A_{2} t+A_{3}\right)^{2}-\left(x-e_{1}\right)\left(x-e_{2}\right)\left(x-e_{3}\right)=0,
$$

in the $x-t$ plane, a description that will be useful later. Since $F$ is always linear in $y$, one can for $n>3$ always solve $F=0$ to give $y$ as a rational function (not a polynomial if $n>3)$ in $x$ and $t$; substituting in the equation $y^{2}=\prod_{i}\left(x-e_{i}\right)$ then exhibits $C$ as a rather complicated curve in the $x-t$ plane.

\subsection{Singularities For Weak Coupling}

Another check comes by comparing to the singularity structure of the theory in the weak coupling limit. Of course, we have already looked at the limit of weak coupling with the $A_{k}$ going to "zero"; now we will look at weak coupling with the $A_{k}$ fixed.

In general, a singularity occurs for values of the $A_{k}$ at which an extra massless particle appears. In the classical limit, the mass spectrum can be read off from the classical 
Lagrangian. If the Higgs field $\phi$ has eigenvalues $a_{1}, \ldots, a_{n}$ (with $\sum_{i} a_{i}=0$ ), then in the classical limit, the masses of the vector supermultiplets that are not massless generically equal $\left|a_{i}-a_{j}\right|$ with $i \neq j$, and the masses of the hypermultiplets are (if the bare mass is 1) $\left|a_{i}-a_{j}-1\right|$. A singularity will arise from a zero of either

$$
D_{V}=\prod_{1 \leq i<j \leq n}\left(a_{i}-a_{j}\right)^{2}
$$

or

$$
D_{H}=\prod_{1 \leq i, j \leq n}\left(a_{i}-a_{j}-1\right)=(-1)^{n(n+1) / 2} \prod_{1 \leq i<j \leq n}\left(\left(a_{i}-a_{j}\right)^{2}-1\right) .
$$

We will study the weak coupling behavior in detail for $n=3$. For $n=3$, if

$$
\begin{gathered}
W_{2}=a_{1} a_{2}+a_{2} a_{3}+a_{3} a_{1} \\
W_{3}=a_{1} a_{2} a_{3}
\end{gathered}
$$

are the elementary symmetric polynomials in the eigenvalues of $\phi$, then one has explicitly

$$
\begin{aligned}
& D_{V}=-4 W_{2}^{3}-27 W_{3}^{2} \\
& D_{H}=-\left(4 W_{2}+1\right)\left(W_{2}+1\right)^{2}-27 W_{3}^{2} .
\end{aligned}
$$

On the other hand, if we denote the left hand side of (3.27) as $Q(x, t)$, for $n=3$ a singularity of $C$ arises precisely when $Q=\partial Q / \partial x=\partial Q / \partial t=0$. The equation $\partial Q / \partial t=0$ gives 10

$$
3 t^{2}-3 x+A_{2}=0
$$

This can be used to eliminate $x$ from (3.27), so we reduce to an equation $H(t)=0$ for some polynomial $H$ :

$$
\begin{aligned}
H= & \left(A_{2}-\sum_{i} e_{i}\right) t^{4} \\
& +A_{3} t^{3}+\left(\frac{1}{3} A_{2}^{2}-\frac{2}{3} A_{2} \sum_{i} e_{i}+\sum_{i<j} e_{i} e_{j}\right) t^{2}-\frac{1}{4} A_{3}^{2}+\frac{1}{27} \prod_{i=1}^{3}\left(A_{2}-3 e_{i}\right) .
\end{aligned}
$$

10 There is another branch $t^{3}-3 x t+A_{2} t+A_{3}=0$, but on this branch it is impossible to satisfy $Q=\partial Q / \partial x=0$ if the $e_{i}$ are distinct. If the $e_{i}$ are not distinct, the singularity that we get when $t^{3}-3 x t+A_{2} t+A_{3}=0$ does not affect the part of the Jacobian of $C$ that is actually relevant to the $N=2$ model - it affects the part that comes from $E$. 
Singularities of the curve $C$ show up as solutions of $H=d H / d t=0$, but the converse is not quite true; the equations $H=d H / d t=0$ can have solutions at $t=0$ that do not come from singularities of $C$. The singular locus of $C$ can be found finally by computing the discriminant of $H(t)$ and throwing away a factor that comes from solutions of $H=$ $d H / d t=t=0$. One is left with a very complicated polynomial $\Delta\left(A_{2}, A_{3}\right)$ which we will call the discriminant.

However, for weak coupling a simplification appears. One can go to weak coupling by setting $e_{i}=(0,0,1)$, and then $\Delta$ turns out to factor as

$$
\left(4 A_{2}^{3}+27 A_{3}^{2}\right)^{2}\left(4\left(A_{2}-1\right)\left(A_{2}-4\right)^{2}+27 A_{3}^{2}\right)
$$

This coincides with $D_{V}^{2} D_{H}$ if we identify

$$
A_{k}=(2 i)^{k} W_{k}
$$

The factor of $(2 i)^{k}$ could be absorbed in rescaling the Higgs field. The fact that $D_{V}$ is squared but $D_{H}$ is raised to the first power presumably has something to do with the fact that extra massless vector multiplets come in pairs, but extra massless hypermultiplets come one at a time. Note that the constants in (3.35) are fixed by recovering the formula for $D_{V}$, and then one automatically gets $D_{H}$.

\subsection{Confinement And Higgs Mechanism For $N=1$}

Now we want to discuss a key point of physics: how one sees confinement and the Higgs mechanism in this formalism.

In [1], the elliptic curve controlling the pure $N=2$ theory for $S U(2)$ developed a node or ordinary double point at certain points in the complex $u$ plane. Under a certain mass perturbation that breaks $N=2$ to $N=1$ supersymmetry, $u$ is locked near one of these points; monopole condensation occurs, giving a mass gap to the $N=2$ theory and triggering confinement.

For groups of rank $r>1$, the theory is described in terms of a Riemann surface of higher genus 11 Every time a node develops, the genus of the Riemann surface drops by one and there appears a massless monopole that is charged under one of the $U(1)$ 's in the low energy gauge group. The massive vacua that will appear in the presence of the

11 Or in general, an abelian variety of higher rank. We will use the Riemann surface language for simplicity and because it is adequate for the present case. 
mass perturbation to $N=1$ - massive because of confinement or a Higgs mechanism correspond to points in moduli space at which there are $r$ different nodes, so that the low energy gauge group is completely broken by monopole condensation.

In our problem, we start with a genus one surface $E$, and then determine the physics by an $n$-fold cover $C \rightarrow E, C$ being a Riemann surface of genus $n$. The low energy gauge group has rank $r=n-1$ (that being the rank of $S U(n)$ ). To get a totally massive vacuum, $C$ must develop $r$ nodes. This will reduce the genus of $C$ to one. The cover $C \rightarrow E$ is still an $n$-fold cover, unramified as $C$ and $E$ both have genus one.

Totally massive vacua are thus associated with $n$-fold unramified covers of $E$; moreover, given such a cover, there is precisely one point in the moduli space of the Coulomb branch at which it occurs. To verify the last assertion, start with an $n$-sheeted cover $\pi: \widetilde{E} \longrightarrow E$. The claim is that there is a unique $C$ of arithmetic genus $n$ with normalization (=desingularization) $\widetilde{E}$, which occurs as a spectral curve in our system. First note that the automorphisms of $\widetilde{E}$ over $E$ permute the $n$ points in $\pi^{-1}(p)$ transitively, so it does not matter which of these we label as the point $q_{0}$ where the residue is $-(n-1)$. Having made that choice, Riemann-Roch guarantees the existence of a unique function $f$ on $\widetilde{E}$ with first order poles at $\left\{q_{0}, q_{1}, \ldots, q_{n-1}\right\}=\pi^{-1}(p)$, and respective residues $-(n-1),+1, \ldots,+1$. The spectral curve $C$ is then essentially the image of $\widetilde{E}$ in $E \times \mathbf{P}^{\mathbf{1}}$ under $(\pi, f)$.

Thus to classify the totally massive vacua is the same as classifying the $n$-fold unramified covers $C \rightarrow E$. This is easily done. One can realize $E$ as $\mathbf{C} / \Gamma$ with $\Gamma \cong \mathbf{Z} \oplus \mathbf{Z}$ a lattice in the complex plane $\mathbf{C}$. In that realization, the $n$-fold cover $C$ is $\mathbf{C} / \Gamma^{\prime}$, where $\Gamma^{\prime}$ is a sublattice of $\Gamma$ of index $n$. Any such sublattice contains $n \Gamma$, which is a sublattice of $\Gamma$ of index $n^{2}$. The quotient $\Gamma / n \Gamma$ is an abelian group isomorphic to $F=\mathbf{Z}_{n} \times \mathbf{Z}_{n}$. The quotient $\Gamma^{\prime} / n \Gamma$ is a subgroup $F^{\prime}$ of $F$ of index $n$. Conversely, every index $n \operatorname{subgroup~} F^{\prime}$ of $F$ determines by this construction a $C$.

So the set $\mathcal{S}$ of massive vacua (in the presence of the perturbation to $N=1$ ) is the same as the set of index $n$ subgroups $F^{\prime}$ of $F=\mathbf{Z}_{n} \times \mathbf{Z}_{n}$. The action of $S L(2, \mathbf{Z})$ on $\mathcal{S}$ comes from its action on $\Gamma$ and thus from the natural action of $S L(2, \mathbf{Z})$ on $\mathbf{Z}_{n} \times \mathbf{Z}_{n}$.

In fact, according to 't Hooft's abstract classification [16] of phases of $S U(n)$ gauge theory, the possible massive phases are classified by index $n$ subgroups of $\mathbf{Z}_{n} \times \mathbf{Z}_{n}$. (This is perhaps not as well known as the principles of the classification of phases and will be explained in section four.) Thus, the result in the last paragraph strongly indicates that this theory (perturbed to $N=1$ ) realizes every possible massive phase of an $S U(n)$ gauge theory precisely once. We will argue this directly in section four (where we will also discuss 
some Coulomb phases of this theory). The relation to 't Hooft's classification also makes it clear that the two factors of $\mathbf{Z}_{n}$ in $F=\mathbf{Z}_{n} \times \mathbf{Z}_{n}$ can be considered as labeling magnetic and electric charge; then the action of $S L(2, \mathbf{Z})$ on $\mathcal{S}$ found in the last paragraph is the expected action of $S$-duality on the magnetic and electric charges in the vacuum condensate.

For $n=2, F$ is equivalent to the additive group $\mathbf{Z}_{2} \times \mathbf{Z}_{2}$ of points of order two in $E$, and the choice of an index two subgroup $F^{\prime}$ is just the choice of a non-zero point of order two. Thus our description of the massive vacua and the $S L(2, \mathbf{Z})$ action on them agrees for $n=2$ with the description in [2], where these vacua were identified with the non-zero points of order two.

\section{Details For $n=3$}

To make this more concrete, we will briefly explain how to explicitly find for $n=3$ the massive vacua, that is the points in moduli space at which $C$ has $n-1=2$ nodes. There are three index three subgroups of $\mathbf{Z}_{3} \times \mathbf{Z}_{3}$ generated by $(1, x)$ for $x=1,2$, or 3 , and one generated by $(0,1)$. In all, then, there should be four values of $A_{2}$ and $A_{3}$ at which $C$ has two nodes. Let us find them.

First of all, these points are all at $A_{3}=0$. (More generally, we will see in section four that the massive vacua are associated with certain $S U(2)$ generators $J$. As $\operatorname{Tr} J^{2 r+1}=0$ for any $S U(2)$ generator, the massive vacua all have $A_{2 r+1}=0$ for any $r$ and $n$.) This being so, equation (3.33) simplifies to

$$
H=\left(A_{2}-\sum_{i} e_{i}\right) w^{2}+\left(\frac{1}{3} A_{2}^{2}-\frac{2}{3} A_{2} \sum_{i} e_{i}+\sum_{i<j} e_{i} e_{j}\right) w+\frac{1}{27} \prod_{i=1}^{3}\left(A_{2}-3 e_{i}\right)
$$

with

$$
w=t^{2}
$$

We recall that singularities of $C$ correspond to solutions of $H=d H / d t=0$ with $t \neq 0$. If we write (3.36) as $\alpha w^{2}+\beta w+\gamma$, then $\alpha, \beta$, and $\gamma$ are respectively linear, quadratic, and cubic in $A_{2}$, then the discriminant $\beta^{2}-4 \alpha \gamma$ is quartic. When the $e_{i}$ are distinct, this quartic function vanishes at four distinct values of $A_{2}$. For such a value of $A_{2}$, the quadratic form in (3.36) vanishes at a unique value of $w$; the two desired nodes of $C$ are then at $t= \pm \sqrt{w}$, with $x$ and $y$ determined by $(3.32)$ and $(3.26)$. Note that this argument actually determines all the singularities at $A_{3}=0$; they are the expected nodes, and nothing else. 


\subsection{Cusps}

A cusp is simply a singularity of a Riemann surface that looks like the singularity of the curve $y^{2}=x^{3}$ at $x=y=0$. A cusp in the Riemann surface governing an $N=2$ supersymmetric system corresponds apparently to a novel and mysterious kind of critical point, as discussed in [18].

Focussing on the case of gauge group $S U(3)$, let us count, in the weak coupling regime, how many cusps can be predicted in our theory because of known physical mechanisms. First of all, there is a region, discussed in section 3.1, in which the theory flows to the pure $S U(3)$ model, without hypermultiplets. This theory has 18 cusps at two values of $A_{2}, A_{3}$. There is also a second regime that one should consider. For this we recall the weak coupling discriminant from section 3.3. The $\lambda=0$ discriminant has a factor $D_{V}$ that vanishes precisely when the theory has an unbroken $S U(2)$ gauge symmetry. It has a factor $D_{H}$ that vanishes precisely when there is a massless hypermultiplet. One finds that $D_{V}=D_{H}=0$ at the two points $A_{2}=4 / 3, A_{3}= \pm 16 i / 27$. Scaling $\lambda$ to zero in a neighborhood of those points, one expects to reduce to the $S U(2)$ theory with a massless hypermultiplet in the two-dimensional representation. $A_{2}$ and $A_{3}$ correspond to the order parameter $u$ and the bare mass in this theory. This model was analyzed in [2] and has cusps at three values of the parameters [26]. Therefore, the model under study here for weak coupling (and therefore generically) should have cusps at at least $2+2 \cdot 3=8$ values of $A_{2}, A_{3}$. It turns out that this is the exact number for any value of $\lambda$ away from $0,1, \infty$.

In searching for cusps at given $\lambda$, it helps to know that the unfolding $y^{2}=x^{3}+\alpha x+\beta$ has the property that the discriminant, as a function of $\alpha$ and $\beta$, has a cusp at $\alpha=\beta=0$. Conversely, cusps in the discriminant reflect cusps in the curve.

So to find cusps in the spectral curve, one can look for cusps in the discriminant curve $\Delta\left(A_{2}, A_{3}\right)=0$, where the discriminant $\Delta$ was defined in section 3.3. Cusps in the discriminant curve can be found by Maple. One way is to first compute the discriminant of $\Delta\left(A_{2}, A_{3}\right)$ regarded as a polynomial in $A_{3}$ with $A_{2}$ as a parameter, and then look for multiple roots of the resulting polynomial $g\left(A_{2}\right)$. It turns out that $g\left(A_{2}\right)=h\left(A_{2}\right)^{3}$ where $h$ is a quartic polynomial whose zeroes are the $A_{2}$ coordinates of the cusps; for each such zero there are two values of $A_{3}$ (related by the symmetry $A_{3} \rightarrow-A_{3}$ ) at which the discriminant curve has a cusp. If we set $a=6 A_{2}$, then up to a constant factor the polynomial $h(a)$ is

$$
\begin{aligned}
h(a)= & a^{4}-24(1+\lambda) a^{3}+\left(192+456 \lambda+192 \lambda^{2}\right) a^{2}-\left(512\left(1+\lambda^{3}\right)+2688 \lambda(1+\lambda)\right) a \\
& +\left(4608 \lambda+8784 \lambda^{2}+4608 \lambda^{3}\right) .
\end{aligned}
$$


As $\lambda \rightarrow 0$, the four roots of this equation behave as follows. One has $a \rightarrow 0$, corresponding to the two cusps of the pure $S U(3)$ theory, and the other three have $a \rightarrow 8$, the value of $a$ for $D_{V}=D_{H}=0$ where one sees the $S U(2)$ theory with a massless doublet hypermultiplet.12

Now we come to an important point. The discriminant of the polynomial $h(a)$ can be readily computed and turns out to be a power of $\lambda(\lambda-1)$. In particular it never vanishes except at $\lambda=0$ or 1 , two points (equivalent under $S L(2, \mathbf{Z})$ ) that correspond to weak coupling or $\tau=i \infty$. Thus, except at infinity in moduli space, the $A_{2}$ values of the four cusp pairs are all distinct. Moreover, the two cusps of given $A_{2}$ are related by $A_{3} \rightarrow-A_{3}$ and are distinct since the cusps never have $A_{3}=0$. (At the end of section 3.4 we analyzed all singularities at $A_{3}=0$ without meeting cusps.) Hence, for any (finite) $\tau$ the eight cusps are all distinct.

This has the following significance: it means that there is a well-defined action of $S L(2, \mathbf{Z})$ on the set of eight cusps. 13 This is analogous to the fact that the massive phases also never meet for finite $\tau$, so that there is an $S L(2, \mathbf{Z})$ action on the set of massive phases, which was determined above. The difference is that the massive phases are more or less understood, and there was a prediction for how $S L(2, \mathbf{Z})$ should act on them; the cusps are not well understood and there is no prediction to compare to.

The result that emerges for the action of $S L(2, \mathbf{Z})$ on the cusps is as follows. Let $u, v$ be integers defined modulo three and not both zero. Note that there are eight possible choices of the pair $u, v$. The natural two-dimensional representation of $S L(2, \mathbf{Z})$ can be reduced mod three to give an action by permutation of the eight possible pairs $u, v, 14$ and we claim that this is the representation by which $S L(2, \mathbf{Z})$ acts on the cusps. Note that this is equivalent to saying that the cusps correspond to points of order three on $E$. Also,

12 Incidentally, beyond locating the cusps, this computation can be extended to give a complete classification of the singularities of the discriminant. There are four nodes corresponding to the massive phases that we discussed above, eight cusps, and a higher singularity, a tacnode, at infinity. We do not know the physical meaning of that latter singularity.

13 Suppose one starts at some point $\tau$ in the upper half plane with a particular cusp $C$. Given $g \in S L(2, \mathbf{Z})$, one wants to know how $g$ acts on $C . g$ naturally maps $C$ to a cusp $g(C)$ in the theory with a different $\tau$-parameter $g(\tau)$. However, by parallel transport in the upper half plane from $g(\tau)$ back to $\tau$, one can identify $g(C)$ with a cusp at $\tau$. Getting an unambiguous answer this way depends on the fact that the cusps never meet; if several cusps were to meet at $\tau=\tau_{0}$, the result of parallel transport would be affected by which way one wraps around $\tau_{0}$.

14 Think of $u, v$ as a mod three column vector $\left(\begin{array}{l}u \\ v\end{array}\right)$ acted on by an $S L(2, \mathbf{Z})$ matrix. 
note that if we divide by the center of $S L(2, \mathbf{Z})$ and identify $u, v$ with $-u,-v$, we would have four equivalence classes corresponding to the four subgroups of order three; these are from the above analysis in one-to-one correspondence with the massive phases.

Though we do not know a natural proof that the $S L(2, \mathbf{Z})$ action on the cusps is as stated above, it is possible to verify this by checking generators and relations. $S L(2, \mathbf{Z})$ is generated by the two elements

$$
\begin{aligned}
S & =\left(\begin{array}{cc}
0 & 1 \\
-1 & 0
\end{array}\right) \\
T & =\left(\begin{array}{ll}
1 & 1 \\
0 & 1
\end{array}\right)
\end{aligned}
$$

with relations $S^{4}=(S T)^{3}=1$ and $S^{2} T=T S^{2}$. Note that $S^{2}$ is the central element -1 of $S L(2, \mathbf{Z})$, which acts by $A_{3} \rightarrow-A_{3}$. We know that -1 does not leave fixed any cusp (since no cusp is at $A_{3}=0$ ), so as a permutation of the eight cusps $S^{2}$ is conjugate to (12)(34)(56)(78). It follows that $S$ is conjugate to (1234)(5678). As for $T$, it is the transformation $\theta \rightarrow \theta+2 \pi$ on the underlying $\theta$ angle. Physically, this is expected to leave invariant the cusps in the pure $S U(3)$ theory while permuting the three cusps in the $S U(2)$ theory with the hypermultiplet. This behavior can also be seen in the monodromy of the four roots of $h(a)$ around $\lambda=0$; one is invariant, and three are permuted. Thus $T$ is conjugate to the permutation 12(345)(678). It is a straightforward exercise to check that there is only one action of $S L(2, \mathbf{Z})$ by permutation of eight objects with the conjugacy classes of $S$ and $T$ as given, so the action of $S L(2, \mathbf{Z})$ on the cusps is as stated in the last paragraph.

Finally, note that the action of $S L(2, \mathbf{Z})$ on the eight cusps is transitive. This in particular proves that the critical point associated with the cusp in the pure $S U(3)$ theory is equivalent to the critical point associated with the cusp in the $S U(2)$ theory with the hypermultiplet.

\subsection{Higgs to Infinity}

As another check of our model, we want to consider the flow from $S U(n)$ to a subgroup as $\phi$ becomes large. If $\phi=B+C / s$, where $C$ is a block-diagonal matrix that breaks $S U(n)$ to a product $H=\prod_{i} S U\left(n_{i}\right) \times U(1)^{k-1}$, where $n_{i}$ are the sizes of the blocks in $C$ and $k$ is the number of blocks, then in the limit of $s \rightarrow 0$, we should reduce to a product of $S U\left(n_{i}\right)$ theories with free $U(1)$ theories. This is actually one point where it is more transparent to use the abstract formulation of section 2 rather than the explicit equations that we have used so far in the present section. 
In section 2 , we had a vector bundle $V$ over an elliptic curve $E$, which decomposes as a sum of line bundles

$$
V=\bigoplus_{i=1}^{n} L_{i}
$$

There was also a differential $\phi$ on $E$, with values in the adjoint representation, and a prescribed type of pole at a distinguished point $p$ in $E ; \phi$ is often called the Higgs field. The decomposition $\phi=B+C / s$ of the four-dimensional Higgs field simply corresponds to a decomposition $\phi=B+C / s$ for the two-dimensional Higgs field, in the following sense. In the basis in (3.40), $C$ is the same block-diagonal matrix with constant diagonal elements that appears in the four-dimensional description, but $B$ is now different - it is an adjointvalued differential on $E$ with a particular sort of pole (whose characteristic polynomial will nonetheless ultimately be related to the vacuum expectation value of the physical Higgs field).

The spectral cover is now given by the equation $\operatorname{det}(t-(B+C / s))=0$; we want to take the limit of the cover as $s$ goes to zero. After an obvious rescaling of $t$, we have to look at the equation $\operatorname{det}(t-C-s B)=0$ in the limit of small $s$. It may appear that the limit is just the equation $\operatorname{det}(t-C)=0$, and this is so if the eigenvalues of $C$ are all distinct, in which case the unbroken group is just $U(1)^{n-1}$. The more interesting case is that in which $C$ has some equal eigenvalues, corresponding to a non-abelian unbroken group. In that case, the minors of $B$ in the blocks in which $C$ is constant cannot be disregarded as they lift the degeneracy otherwise present. Letting $C_{i}$ be the value of $C$ in the $i^{\text {th }}$ block, and $B_{i}$ the corresponding minor of $B$, and letting $t-C_{i}=s \tilde{t}$, the equation in the $i^{t h}$ block becomes $\operatorname{det}\left(\widetilde{t}-B_{i}\right)=0$. Since $B_{i}$ is not necessarily traceless, this is the spectral cover for the $U\left(n_{i}\right)$ theory; its Jacobian can be decomposed as the product of the $S U\left(n_{i}\right)$ abelian variety and a factor of $E$ for each $U(1)$.

There is actually one more point to clarify, which is that $B_{i}$ automatically has a residue of the right sort. That is because the residue condition was that the residue of $\phi$ was diagonalizable and equal to 1 plus a matrix of rank 1, a condition inherited by any generic minor such as $B_{i}$. Note that the residue of $B_{i}$ is automatically traceless, as the trace of $B_{i}$ is an ordinary differential on $E$ with at most a single pole at $p$; the pole must be absent as the residues of a differential form on $E$ always sum to zero. 


\subsection{The Surface}

In this section we discuss the symplectic form on the integrable system. First we give a fairly explicit construction of this form and discuss its linear dependence on parameters in our setup, and then we place this in a broader context by reviewing some of the relevant mathematics literature.

The construction has two parts. The symplectic form on the cotangent bundle $T^{*} E=$ $E \times \mathbf{C}$ induces a two-form on the total space of the family of spectral curves. This in turn determines a two-form on the total space of the family of Jacobians of spectral curves, which is the total space of the integrable system, as was explained in section 2.4. We begin with this latter step.

Start with any family $C_{b}, b \in B$ of curves, parametrized by some base $B$, and let $\mathcal{C}$ be the total space of the family. Let $J_{b}$ be the Jacobian of $C_{b}$, let $\mathcal{J}$ be the total space of the family of Jacobians, and let $\mathcal{C}^{(n)}$ be the relative $n$-th symmetric product of $\mathcal{C}$, that is, the total space of the family over $B$ whose fiber at $b \in B$ is the $n$-th symmetric product $C^{(n)}$ of $C$. A two-form $\sigma$ on $\mathcal{C}$ determines, in a natural way, a two-form $\tau$ on $\mathcal{J}$. One way to see this is to note that the Abel-Jacobi map sends $\mathcal{C}$, as well as the various $\mathcal{C}^{(n)}$, to $\mathcal{J}$. It is clear how to use $\sigma$ to build a two-form on $\mathcal{C}^{(n)}$; but for $n>>0$, the Abel-Jacobi map $\mathcal{C}^{(n)} \longrightarrow \mathcal{J}$ is a fiber bundle with projective spaces for fibers, so the form on $\mathcal{C}^{(n)}$ must be the pullback of one on $\mathcal{J}$. (One needs to check that the result is independent of the choice of base point for the Abel-Jacobi map.)

If $\mathcal{C}$ happens to be given as a family of curves on a symplectic surface $S$, there is a natural two-form $\sigma$ on $\mathcal{C}$ obtained by pulling back the symplectic form via the projection $\operatorname{map} \mathcal{C} \longrightarrow \mathcal{B}$. This is the case for Hitchin's system, where the surface is the cotangent bundle $T^{*} E$, and the symplectic form on it is $d x \wedge d t / y$. (Here $x, y$ are the usual functions on $E$, and $t$ is the vertical coordinate, on C.) In our case things are slightly more complicated, since the spectral curves are not contained in $T^{*} E=E \times \mathbf{C}$ but rather in its compactification $T:=E \times P^{1}$. The form $d x \wedge d t / y$ is meromorphic on $T$, with second order pole along $\{t=\infty\}=E \times \infty$. The spectral curves intersect the polar locus only at the point $\{t=\infty, x=\infty\}$, but in a rather complicated way; so it is natural to transform them to another surface $S$ which is birationally equivalent to $T$. Specifically, we take $S$ to be the $P^{1}$-bundle over $E: S:=\mathbf{P}\left(O_{E}+O_{E}(\infty)\right)$. It can be obtained from $T=\mathbf{P}\left(O_{E}+O_{E}\right)$ by blowing up the problematic point $\{t=\infty, x=\infty\}$, then blowing down the original fiber over $\{x=\infty\}$. When we map the spectral curves to $S$, they completely miss the section at 
$\infty$, and so are contained in the affine part of $S$, which is the total space of the line bundle $O_{E}(\infty)$. For the region of this affine surface near the fiber over $x=\infty$, the natural coordinates arising from the blowup are $u$ and $s:=t u$, where $u$ is the coordinate at infinity on $E$ used before (so $x=u^{-2}$ and $y=u^{-3}+\ldots$ ). The two-form is $d x \wedge d t / y=\left(-2 u^{-4} / y\right) d u \wedge d s$, so it has a first-order pole along $u=0$ (as well as a second order pole along $s=\infty$, but our spectral curves never meet this latter locus). Nevertheless, we claim that the pullback $\sigma$ of this two-form is everywhere holomorphic, so we can apply the previous construction to get a symplectic form on the family of Jacobians. Indeed, consider the pullback, from a surface $S$ with local coordinates $s, u$ to a manifold $\mathcal{C}$ mapping to it, of a meromorphic two-form $d u \wedge d s / u$ with first-order poles along $u=0$. The pullback is holomorphic if the map is ramified above the polar locus $u=0$. Back in our situation, the equation of the universal spectral curve,

$$
t^{n}-\frac{n(n-1)}{2} x t^{n-2}+\ldots+A_{2} t^{n-2}+\ldots=0,
$$

when multiplied by $u^{n}$, becomes

$$
s^{n}-\frac{n(n-1)}{2} s^{n-2}+\ldots+A_{2} s^{n-2} u^{2}+\ldots=0 .
$$

Choose any of the $n-1$ sheets near $u=0, s=1$ (or the one near $u=0, s=1-n$ ). The map which projects this to the $(s, u)$-plane is indeed ramified above $u=0$, since the coefficients of each of the $A_{i}$ vanish to order $\geq 2$. We conclude that the pullback $\sigma$ is holomorphic on $\mathcal{C}$, and we get our desired symplectic form on $\mathcal{J}$.

In the above construction, we fixed the mass at $m=1$. Consider what happens when we allow $m$ to vary. The surfaces $T$ and $S$ are fixed, but the family of curves in $S$ appears to depend on $m$. This dependence can of course be eliminated by applying to $S$ the automorphism $s \rightarrow m s$. The price we pay is that the two-form $d x \wedge d t / y$ is now multiplied by $m$. Since the two-forms $\sigma$ and $\tau$ on the respective total spaces of spectral curves and spectral Jacobians are determined from this by linear operations, we conclude that $\tau$ depends linearly on $m$ as claimed.

The existence of families of symplectic forms depending on linear parameters is known to extend in several directions. There is a beautiful result of Mukai [27], which says that the moduli space of simple sheaves on a symplectic surface itself carries a natural symplectic structure. Mukai applies his construction to the moduli of sheaves on an Abelian or K3 
surface, but with a small modification it applies also to Hitchin's system, and with some more significant changes, it turns out to apply to our system as well.

Mukai's result applies to coherent sheaves on a symplectic surface $S$, whose support could be the entire surface (e.g. vector bundles on the surface), or a finite subscheme (this recovers Beauville's construction of a symplectic structure on an appropriate resolution of singularities of the symmetric product of a K3 surface), or as in our case, a curve on the surface. The result is quite intuitive in the case of finite support: the symplectic form on $S$ induces one on the product $S^{n}$, which clearly descends to a symplectic form on a dense open subset of the symmetric product; the only remaining issue is to check that this extends symplectically (i.e. is nowhere degenerate) on a particular compactification of this open subset, which turns out to be the Hilbert scheme parametrizing appropriate sheaves on $S$. In the general case, Mukai identifies the tangent space to moduli at a simple sheaf $F$ with the vector space

$$
\operatorname{Ext}_{O_{S}}^{1}(F, F)
$$

and notes that any two-form $\sigma \in H^{0}\left(\omega_{S}\right)$ determines an alternating bilinear map:

$$
\operatorname{Ext}_{O_{S}}^{1}(F, F) \times \operatorname{Ext}_{O_{S}}^{1}(F, F) \rightarrow \operatorname{Ext}_{O_{S}}^{2}(F, F) \rightarrow H^{2}\left(O_{S}\right) \rightarrow H^{2}\left(\omega_{S}\right) \approx \mathbf{C}
$$

hence a two-form on moduli, which is non-degenerate for general duality reasons. In general, one still needs to check that this form is closed; when the moduli space is known to be smooth and projective, this follows for free from the Kahler package.

Hitchin's system fits as the special case where the surface is the cotangent bundle $T^{*} E$ of a curve $E$, and the sheaves are (the extension to the surface of) line bundles on (spectral) curves. In our case, the sheaves are supported on curves contained in the surface $S$ which is the total space of the non-trivial line bundle $O_{E}(p)$, and this surface is not symplectic. Rather, it is Poisson, i.e. it has a natural two-vector field (the inverse of the meromorphic two-form $\left.d x \wedge d t / y=\left(-2 u^{-4} / y\right) d u \wedge d s\right)$ which is non-degenerate only generically, away from $u=0$. Now a modification of Mukai's argument (observed by Tyurin, Mukai, and Markman) shows that the moduli of sheaves on a Poisson surface itself carries a Poisson structure. The space we are interested in then appears as one symplectic leaf of this general Poisson space (or as a one-parameter family of such leaves, if we allow the mass to vary rather than fixing it at 1$)$. 


\section{Analysis Of The Massive Phases}

This final section is devoted mainly to explaining some points of physics that are needed to properly understand the analysis of the totally massive vacua given in section three. We will also make a few remarks on Coulomb phases.

We first make a few simple observations on 't Hooft's abstract classification of the possible vacua. Then we explain from a weak coupling point of view why the particular theory under study has the beautiful property of realizing each of these vacua precisely once. Then we turn to the Coulomb phases.

\subsection{Classification Of Phases}

Consider an $S U(n)$ gauge theory in which all fields transform trivially under the center of $S U(n)$, which is isomorphic to $\mathbf{Z}_{n}$. An example is the perturbed $N=4$ theory discussed in this paper. One might say that in such a theory the gauge group could really be taken as $S U(n) / \mathbf{Z}_{n}$.

The group of possible external charges by which this theory might be probed, modulo the charges of the dynamical fields, is the group of characters of $\mathbf{Z}_{n}$ and is isomorphic to $\mathbf{Z}_{n}$. We refer to this $\mathbf{Z}_{n}$ as the group of electric charges.

In addition, if one is in four dimensions, then as the fundamental group of $S U(n) / \mathbf{Z}_{n}$ is again $\mathbf{Z}_{n}$, the possible $S U(n) / \mathbf{Z}_{n}$ bundles on a two-sphere at spatial infinity correspond to elements of $\mathbf{Z}_{n}$. This $\mathbf{Z}_{n}$ classifies the possible magnetic charges by which the theory can be probed. The group $F=\mathbf{Z}_{n} \times \mathbf{Z}_{n}$ of these possible magnetic and electric charges (we will write the magnetic charge first and the electric charge second) plays a fundamental role in 't Hooft's abstract classification of the possible phases of an $S U(n)$ gauge theory.

For every point $(a, b) \in F$, and every loop $C$ in space-time, one can define a loop operator $W_{a, b}(C)\left(W_{(0,1)}(C)\right.$ is the Wilson loop operator, and $W_{(1,0)}(C)$ is often called the 't Hooft loop operator; $W_{a, b}(C)$ is essentially the product of $a$ copies of the 't Hooft operator and $b$ copies of the Wilson operator). In computing the algebra of the $W$ 's, one meets a certain natural skew form $\langle$,$\rangle on F$, with values in $\mathbf{Z}_{n}$. $\langle$,$\rangle can be defined by$ saying that for $x=(a, b)$ and $y=(c, d)$,

$$
\langle x, y\rangle=a d-b c \text { modulo } n \text {. }
$$

The importance of this skew form in the theory begins with the following. If a field of charge $x$ condenses in the vacuum, then (as 't Hooft argues) any field $y$ with $\langle x, y\rangle \neq 0$ is 
confined. Therefore, confinement of electric charge follows from condensation of magnetic charge, and vice-versa. Of course, this generalizes the usual Meissner effect in superconductors.

't Hooft further proves that if charges $x$ and $y$ both condense, then $\langle x, y\rangle=0$. It follows immediately that if $F^{\prime}$ is the group generated by the condensed charges, then the order $d$ of $F^{\prime}$ is at most $n$, and in fact $d$ is a divisor of $n$. (Conversely, every $F^{\prime}$ whose order is a divisor of $n$ automatically has the property that $\langle x, y\rangle=0$ for $x, y \in F^{\prime}$. For $F^{\prime}$ of order $n$ this follows from the classification of the index $n$ subgroups below; the general case is similar.)

In this situation, let $F^{\prime \prime}$ be the group consisting of all $y$ such that $\langle x, y\rangle=0$ for all $x \in F^{\prime}$. Then $F^{\prime \prime}$ contains $F^{\prime}$, and $F^{\prime \prime}$ is equal to $F^{\prime}$ if and only if the order of $F^{\prime}$ is $n$. In fact, the order of $F^{\prime \prime}$ is $n^{2} / d$, and the index of $F^{\prime}$ in $F^{\prime \prime}$ is $(n / d)^{2}$. Let $L=F^{\prime \prime} / F^{\prime}$. Since the unconfined charges are those in $F^{\prime \prime}$, and the charges in $F^{\prime}$ have condensed in the vacuum, $L$ classifies the unconfined external charges by which the low energy theory can be probed.

For the $L$ equivalence classes of low energy charges, one can still use the 't HooftWilson loop operators $W_{a, b}(C)$. They obey the same algebra as before, still governed by the pairing in (4.1), except that since we are only measuring the charges modulo $F^{\prime}$, the pairing is now well-defined only modulo $n / d$.

't Hooft, however, proves that as long as there are such non-trivial pairings observed in the low energy theory, there cannot be a mass gap; one necessarily has a "Coulomb phase." The massive vacua (that is, the vacua with a mass gap) therefore have $d=n$; they are classified by the order $n$ subgroups $F^{\prime}$ of $F=\mathbf{Z}_{n} \times \mathbf{Z}_{n}$. Any operation that one would want to call electric-magnetic duality should be a subgroup of $S L(2, \mathbf{Z})$ that permutes the massive vacua through the natural action of $S L(2, \mathbf{Z})$ on $\mathbf{Z}_{n} \times \mathbf{Z}_{n}$.

This is precisely the structure that we found in section three: the massive vacua of our theory are classified by index $n$ subgroups of $\mathbf{Z}_{n} \times \mathbf{Z}_{n}$, permuted in the natural way by $S L(2, \mathbf{Z})$. This strongly suggests that each and every massive phase allowed abstractly is realized precisely once in the theory that we are studying. We will give further evidence for that interpretation below.

Before doing so, however, let us make a few simple observations on the index $n$ subgroups of $\mathbf{Z}_{n} \times \mathbf{Z}_{n}$. For $n$ prime, there are precisely $n+1$ of these, generated by $(1, x)$ for some $x \in \mathbf{Z}_{n}$ or by $(0,1)$. (In the present context, condensation of $(1, x)$ corresponds to confinement or oblique confinement, while condensation of $(0,1)$ is the Higgs mechanism.) 
If $n$ is not prime, the structure is more complicated. For every positive divisor $d$ of $n$, and every $b$ with $0 \leq b \leq d-1$, there is an index $n$ subgroup of $\mathbf{Z}_{n} \times \mathbf{Z}_{n}$ generated by $x=(n / d, b)$ and $y=(0, d)$. It is easy to show that all the index $n$ subgroups are of this form for some $d$ and $b, 15$ so the number of possible massive phases is the sum of the positive divisors of $n$.

\subsection{Weak Coupling Analysis}

Now we turn to the weak coupling analysis of our particular theory - the $N=2$ theory with a massive matter hypermultiplet. From an $N=1$ point of view, this theory contains an adjoint superfield $\phi$ related by $N=2$ to the gauge field, and two additional adjoint superfields $B, C$ in the hypermultiplet. The superpotential of the $N=2$ theory (including the bare mass term for the hypermultiplet) is

$$
W=\operatorname{Tr}(\phi[B, C]+B C) .
$$

An $N=1$ theory that has massive phases is obtained if one perturbs this by an arbitrarily small mass term for $\phi$. The superpotential is then

$$
W=\operatorname{Tr}\left(\phi[B, C]+B C+\epsilon \phi^{2}\right)
$$

for some complex $\epsilon \neq 0$.

To find the classical vacua, one must find the critical points of $W$ modulo $S L(n, \mathbf{C})$, a task which is invariant under the action of $G L(3, \mathbf{C})$ on the three objects $\phi, B, C$ and under rescaling of $W$ by a constant. Via such operations, one can convert the superpotential into

$$
W=\operatorname{Tr}\left(\frac{1}{2}\left(X^{2}+Y^{2}+Z^{2}\right)-X[Y, Z]\right) .
$$

The equations for a critical point are now

$$
\begin{array}{r}
{[X, Y]=Z} \\
{[Y, Z]=X} \\
{[Z, X]=Y .}
\end{array}
$$

15 Let $F^{\prime}$ be a subgroup of $\mathbf{Z}_{n} \times \mathbf{Z}_{n}$ of order $n$. Let $H$ be the subgroup of $\mathbf{Z}_{n}$ consisting of all $a$ such that $(a, b) \in F^{\prime}$ for some $b$. If $H$ is of order $d$, then $d$ is a divisor of $n$ and $H$ is generated by $n / d \in \mathbf{Z}_{n}$. If so, then $x=(n / d, b) \in F^{\prime}$ for some $b$. For $F^{\prime}$ to have order $n$, the kernel of the homomorphism $F^{\prime} \rightarrow H$ must have order $n / d$, and this means that $F^{\prime}$ must contain $y=(0, d)$. We can therefore assume that $F^{\prime}$ contains $x=(n / d, b)$ with some $b \leq d-1$, as desired. Note that $d$ can be described as the smallest integer such that $(0, d) \in F^{\prime}$. 
As was noted in essentially the present context in [15], these equations are the commutation relations of the Lie algebra of $S U(2)$. Thus, after dividing by $S L(n, \mathbf{C})$, the vacua are parametrized by the isomorphism classes of homomorphisms $\rho$ from $S U(2)$ to $S U(n)$. Since such homomorphisms have no infinitesimal deformations, in any such vacuum, the chiral superfields all have bare masses; any massless particles come from the gauge multiplets.

Given $X, Y$, and $Z$ determining a homomorphism $\rho: S U(2) \rightarrow S U(n)$, the $S U(n)$ gauge group is spontaneously broken at the classical level to the subgroup $H$ that commutes with the image of $\rho$. Since the chiral superfields are massive, the low energy theory is the pure $N=1$ gauge theory with gauge group $H$. If $H$ is semi-simple, confinement will occur at low energies, and one will get a totally massive phase. On the other hand, if $H$ contains $U(1)$ factors, then one will have a Coulomb phase (for these $U(1)$ 's) at low energies, and there will be no mass gap.

$\rho$ defines an $n$-dimensional representation of $S U(2)$ (possibly trivial) which can be decomposed as a sum of irreducible pieces. If there are distinct irreducible pieces in this representation, then $H$ will contain $U(1)$ factors and there will be no mass gap. A mass gap will occur precisely when $\rho$ is the direct sum of $d$ copies of the $n / d$-dimensional irreducible representation of $S U(2)$, and then $H=S U(d)$. The $N=1 S U(d)$ gauge theory without chiral matter has $d$ vacua. Here $d$ can be an arbitrary positive divisor of $n$. So - with every such positive divisor $d$ contributing $d$ vacua - the number of massive vacua in this theory is the sum of the positive divisors of $n$.

Since this coincides with the total number of massive phases possible in $S U(n)$ gauge theory, as counted above, it is natural to think that what is happening in this theory is that each massive phase is appearing precisely once. To justify this, note that in a phase that is Higgsed at the classical level to $H=S U(d)$, an electric charge $(0, c)$ is unconfined (and so is contained in $F^{\prime}$ ) if and only if it transforms trivially under the center of $H$, that is if and only $c$ is divisible by $d$. So in view of the classification of $F^{\prime}$ given in a footnote above, $F^{\prime}$ is generated by $(0, d)$ and $(n / d, b)$, with some $0 \leq b \leq d-1$. (Note that the fundamental magnetic charge of the $S U(d)$ theory corresponds to magnetic charge $n / d$ in $S U(n)$, because of the way $S U(d)$ is embedded in $S U(n)$.) As the symmetry $\theta \rightarrow \theta+2 \pi$ of the low energy $S U(d)$ theory maps $(n / d, b)$ to $(n / d, b+1)$, each value of $b$ appears, and therefore every abstractly possible massive phase is concretely realized in our theory. 


\subsection{Coulomb Phases Of The $N=1$ Theory}

We have analyzed in some detail the massive phases that appear in the theory perturbed by the $\operatorname{Tr} \phi^{2}$ superpotential, and located them in the exact analysis of section three. Coulomb phases are, however, also of interest, and one would like to compare the semiclassical analysis of Coulomb phases to the exact description. We will do this only for $n=3$. (For $n=2$ there are no Coulomb phases after perturbation by $\operatorname{Tr} \phi^{2}$.)

For $n=3, H$ fails to be semisimple only when $\rho$ is the direct sum of a two-dimensional representation of $S U(2)$ and a trivial representation. There is therefore precisely one vacuum of the perturbed theory in a Coulomb phase. Let us try to locate it in the exact solution.

In the exact solution, there is a divisor $D$ in moduli space - one might call it the quantum discriminant - on which a massless charged hypermultiplet appears. Because of reasoning explained in [1], any vacuum that survives when the perturbation $\operatorname{Tr} \phi^{2}$ is turned on is located somewhere on $D$. As sketched in section 3.5, singularities of $D$ are either normal crossings (where the curve $C$ has two nodes) or cusps. The normal crossings give the massive vacua, and [3] the cusps do not survive when the $\operatorname{Tr} \phi^{2}$ perturbation is added. We are therefore dealing with a vacuum associated with a smooth point of $D$.

$D$ can be described as in section 3.3 by an explicit equation $\Delta\left(A_{2}, A_{3}\right)=0$. At a smooth point of $D$, only one monopole hypermultiplet - described in an $N=1$ language by a pair of chiral superfields $M, \widetilde{M}$ - is relevant in the low energy description. The superpotential is thus $W=\Delta\left(A_{2}, A_{3}\right) M \widetilde{M}$. In the presence of the perturbation, since $\operatorname{Tr} \phi^{2}$ is simply a multiple of $A_{2}$, we need to look at the superpotential

$$
W=\Delta\left(A_{2}, A_{3}\right) M \widetilde{M}+\epsilon A_{2}
$$

The equations for a critical point of $W$ are

$$
\begin{aligned}
\Delta\left(A_{2}, A_{3}\right) M=\Delta\left(A_{2}, A_{3}\right) \widetilde{M} & =0 \\
\frac{\partial \Delta}{\partial A_{2}} M \widetilde{M}+\epsilon & =0 \\
\frac{\partial \Delta}{\partial A_{3}} M \widetilde{M} & =0 .
\end{aligned}
$$

The second equation forces $M \widetilde{M} \neq 0$, so the other equations imply

$$
\Delta=\frac{\partial \Delta}{\partial A_{3}}=0
$$


Suppose that by adjusting $A_{2}, A_{3}$ one finds an isolated, nondegenerate solution of those two equations. Assuming that $\partial \Delta / \partial A_{2} \neq 0$ (otherwise one is at a singularity of the discriminant, a case that we have excluded), the second equation in (4.7) then determines the gauge-invariant product $M \widetilde{M}$, which vanishes as $\epsilon \rightarrow 0$. After setting to zero the $D$ terms of the low energy theory and dividing by the gauge group, one gets precisely one vacuum from each isolated solution of (4.8) with $\partial \Delta / \partial A_{2} \neq 0$.

There should be for generic coupling precisely one value of $A_{2}$ and $A_{3}$ obeying these conditions, since we have seen semiclassically that the theory with $\epsilon \neq 0$ has precisely one Coulomb vacuum. To exhibit the existence of such a state, look at the discriminant in the weak coupling limit. This was found in (3.34) to be

$$
\Delta=\left(4 A_{2}^{3}+27 A_{3}^{2}\right)^{2}\left(4\left(A_{2}-1\right)\left(A_{2}-4\right)^{2}+27 A_{3}^{2}\right) .
$$

We notice that the conditions $\Delta=\partial \Delta / \partial A_{3}=0, \partial \Delta / \partial A_{2} \neq 0$ are obeyed precisely at $A_{3}=0, A_{2}=1$. Moreover, at this value of $A_{2}$ and $A_{3}$, the functions $\Delta$ and $\partial \Delta / \partial A_{3}$ vanish only to first order, so if $\Delta$ is perturbed, there will be a nearby solution of these equations at which $\partial \Delta / \partial A_{2}$ will be still non-zero. Thus, we have found a Coulomb vacuum in the exact solution. 


\section{References}

[1] N. Seiberg and E. Witten, "Electric-Magnetic Duality, Monopole Condensation, And Confinement In $N=2$ Supersymmetric Yang-Mills Theory," Nucl. Phys. B426 (1994) 19.

[2] N. Seiberg and E. Witten, "Monopoles, Duality, And Chiral Symmetry Breaking In $N=2$ Supersymmetric QCD," Nucl. Phys. B426 (1994) 484.

[3] P. Argyres and A. Faraggi, "The Vacuum Structure And Spectrum of $N=2$ Supersymmetric $S U(N)$ Gauge Theory," Phys. Rev. Lett. 74 (1995) 3931.

[4] A. Klemm, W. Leche, S. Theisen, and S. Yankielowicz, "Simple Singularities And $N=2$ Supersymmetric Yang-Mills Theory," Phys. Lett. B344 (1995) 169.

[5] U. H. Danielsson and B. Sundborg, "The Moduli Space And Monodromies Of $N=2$ Supersymmetric $S O(2 r+1)$ Yang-Mills Theory," hepth-9504102.

[6] A. Brandhuber and K. Landsteiner, "On The Monodromies Of $N=2$ Supersymmetric Yang-Mills Theory With Gauge Group SO(2n), hepth-9507008.

[7] A. Hanany and Y. Oz, "On The Quantum Moduli Space Of $N=2$ Supersymmetric $S U\left(N_{c}\right)$ Gauge Theories," hepth-9505075.

[8] P. Argyres, R. Plesser, and A. Shapere, "The Coulomb hase Of $N=2$ Supersymmetric QCD," hepth-9505100.

[9] P. C. Argyres and A. D. Shapere, "The Vacuum Structure Of $N=2$ Super-QCD With Classical Gauge Groups," hep-th/9509175.

[10] A. Hanany, "On The Quantum Moduli Space Of Vacua of $N=2$ Supersymmetric Gauge Theories," hep-th/9509176.

[11] N. Hitchin, Stable Bundles and Integrable Systems, Duke Math. J. 54 (1987) 91-114.

[12] E. Markman, Spectral Curves and Integrable Systems, Comp. Math. 93 (1994) 255290.

[13] R. Donagi and E. Markman, "Spectral curves, algebraically completely integrable Hamiltonian systems, and moduli of bundles," 1993 CIME lecture notes, alg-geom9507017, to appear in LNM.

[14] C. Montonen and D. Olive, "Magnetic Monopoles As Gauge Particles?" Phys. Lett. 72B (1977) 177.

[15] C. Vafa and E. Witten, "A Strong Coupling Test Of $S$ Duality," Nucl. Phys. B431 (1994) 3 .

[16] G. 't Hooft, "On The Phase Transition Towards Permanent Quark Confinement," Nucl. Phys. B138 (1978) 1, "A Property Of Electric And Magnetic Flux In Nonabelian Gauge Theory," Nucl. Phys. B153 (1979) 141, "Topology Of The Gauge Condition And New Confinement Phases In Nonabelian Gauge Theories," Nucl. Phys. B190 (1981) 455 . 
[17] J. L. Cardy and E. Rabinovici, "Phase Structure of $Z(p)$ Models In The Presence Of A Theta Parameter," Nucl. Phys. B205 (1982) 1.

[18] P. Argyres and M. Douglas, "New Phenomena In $S U(3)$ Supersymmetric Gauge Theory," hepth-9503163.

[19] E. Martinec and N. Warner, "Integrable Systems and Supersymmetric Gauge Theory," hep-th/9509161.

[20] E. Witten, "Quantum Background Independence in String Theory," hepth-9306122, in Salamfest 1993.

[21] R. Donagi and E. Markman, "Cubics, Calabi-Yau threefolds, Integrable Systems and Mirrors," alg-geom-9408004, to appear in Proc. Hirzebruch Conf., Israel Math. Conf. Proc. (AMS).

[22] J. J. Duistermaat and G. J. Heckman, "On The Variation In The Cohomology In The Symplectic Form Of The Reduced Phase Space," Invent. Math. 69 (1982) 259.

[23] E. Witten, "Phases Of $N=2$ Models In Two Dimensions," Nucl. Phys. B403 (1993) 159.

[24] A. Treibich, J.-L. Verdier, Solitons elliptiques, The Grothendieck Festschrift, Vol. III, 437-480, Birkhauser Boston, 1990.

[25] R. Donagi, "Spectral Covers," alg-geom-9505009, to appear in the proceedings of the MSRI special year in algebraic geometry (1992-93).

[26] P. C. Argyres, M. R. Plesser, N. Seiberg, and E. Witten, "New $N=2$ Superconformal Field Theories In Four Dimensions," to appear.

[27] S. Mukai, Symplectic Structure of the Moduli Space of Sheaves on an Abelian or K3 Surface, Invent. Math. 77 (1984) 101-116 